\title{
Overall Sensitivity Analysis Utilizing Bayesian Network for the Questionnaire Investigation on SNS
}

\author{
Tsuyoshi Aburai $\uparrow$ \\ Graduate School of Policy and Management, \\ Doshisha University, Kyoto, Japan
}

\author{
Kazuhiro Takeyasu \\ College of Business Administration, Tokoha University, \\ Shizuoka, Japan
}

- Structural Equation Modeling requires normal distribution to the data in the analysis. Therefore, it has a limitation in making analysis, but the Bayesian network does not require a specific distribution type to the data. It can handle any distribution type.

- It can handle the data which include partial data.

- Expert's know-how can be reflected in building a Bayesian Network model.

- Sensitivity analysis can be easily performed by settling evidence. We can estimate and predict the prospective purchaser by that analysis.

- It is a probability model having a network structure. Related items are connected with directional link. Therefore, understanding becomes easy by its visual chart.

This research utilizes the Bayesian network to analyze SNS users' current usage conditions, issues and desired functions because no variable is required to have normal distribution. Reviewing past researches, there are some related researches as follows. Tsuji et al. have analyzed preference mining on future home energy consumption [4] [5]. There are some papers concerning purchase behavior in the shop [6] [7], but no research has been reported on the SNS users utilizing Bayesian network.

Bayesian network is utilized for this analysis. Sensitivity analysis is carried out by setting evidence to all items. This enables overall analysis for each item. After conducting the sensitivity analysis, useful results are obtained. Differences in usage objectives and SNS sites are made clear by the attributes and preference of SNS users. It can be utilized effectively for marketing by clarifying the target customer through the sensitivity analysis.

The rest of the paper is organized as follows. The outline of questionnaire research is stated in Section 2. In Section 3, Bayesian network analysis is carried out which is followed by the sensitivity analysis in Section 4. Section 5 is a summary.

\section{OUtLINE Of QuestionnAIRE RESEARCH AND EXAMINEES}

For these purposes, we created a questionnaire investigation of jewelry/accessory purchasing (SNS). In recent years, the Bayesian network is highlighted because it has the following good characteristics [2] [3].

\section{A. Outline of Questionnaire Research}

We make a questionnaire investigation concerning the SNS. Outline of questionnaire research is as follows. 
1)Scope of investigation : student, government employee, and company employee, etc., Japan
2)Period
: April/26/2012-June/6/2012
3)Method
: mail, online and self-writing
4)Collection
: number of distribution 1,500; number of collection 1,197 (collection rate $79.8 \%$ ); Valid answer 1,098 .

\section{B. Outline of Examinees}

We show major single variable summary results in Table 1.

TABLE I. MAJOR SingLE VARIABLE SUMMARY RESUlTS

\begin{tabular}{|c|c|}
\hline Questionnaire & No. of answer (\%) \\
\hline \multicolumn{2}{|l|}{ Q1. Use the SNS } \\
\hline Use & $792(72.1)$ \\
\hline Do not use & $306(27.9)$ \\
\hline \multicolumn{2}{|l|}{ Q13. Gender } \\
\hline Male & $650(59.2)$ \\
\hline Female & $448(40.8)$ \\
\hline \multicolumn{2}{|l|}{ Q14. Age } \\
\hline$<20$ & $196(17.9)$ \\
\hline $21-30$ & $328(29.9)$ \\
\hline $31-40$ & $299(27.2)$ \\
\hline $41-50$ & $194(17.7)$ \\
\hline $51-60$ & $73(6.6)$ \\
\hline$>60$ & $8(0.7)$ \\
\hline \multicolumn{2}{|l|}{ Q15. Occupation } \\
\hline Student & $295(26.9)$ \\
\hline Government employee & $15(1.4)$ \\
\hline Company employee & $595(54.2)$ \\
\hline School teacher/staff & $43(3.9)$ \\
\hline Clerk of organization & $19(1.7)$ \\
\hline Independents & $45(4.1)$ \\
\hline Temporary employee & $15(1.4)$ \\
\hline Part-timers & $53(4.8)$ \\
\hline Miscellaneous & $18(1.6)$ \\
\hline \multicolumn{2}{|l|}{ Q16. Residence } \\
\hline Hokkaido & $22(2.0)$ \\
\hline Tohoku region & $49(4.5)$ \\
\hline Kanto region & $157(14.3)$ \\
\hline Chubu region & $176(16.0)$ \\
\hline Kansai region & $400(36.4)$ \\
\hline Chugoku region & $110(10.0)$ \\
\hline Shikoku region & $105(9.6)$ \\
\hline Kyushu region & $79(7.2)$ \\
\hline
\end{tabular}

\section{BAYESIAN NETWORK ANALYSIS}

In constructing Bayesian network, it is required to set an outline of the model reflecting the causal relationship among groups of items. Concept chart in this case is exhibited in Figure 1.

Haga and Motomura restricted the range of search to the following 5 stages while building the model [8].

(1) Selection of variables

(2) Grouping the variables
(3) Setting the search range for variable groups

(4) Setting the search range within the variable group

(5)Building the total structure

She found that it makes possible to interpret the model easily and to forecast the future activities of variables effectively.

We refer to this sample and build a model where cause and effect relationship is assumed by the order of (I) Purchaser $\Rightarrow$ (II) Extroversion and Usage condition $\Rightarrow$ (III) Purpose for Usage $\Rightarrow$ (IV) SNS. This means that (III) Purpose for Usage for (IV) SNS is influenced by (II) Extroversion and Usage condition, and one's sense of value for these is influenced by the (I) Purchaser.

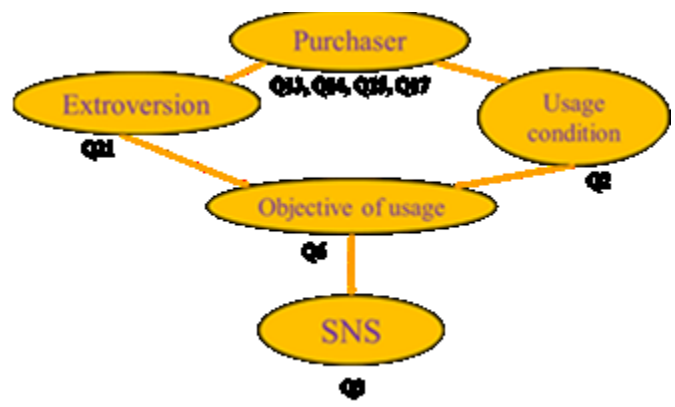

Fig. 1. Node and parameter (source: Takahashi et al, 2008 ; revised by the writer)

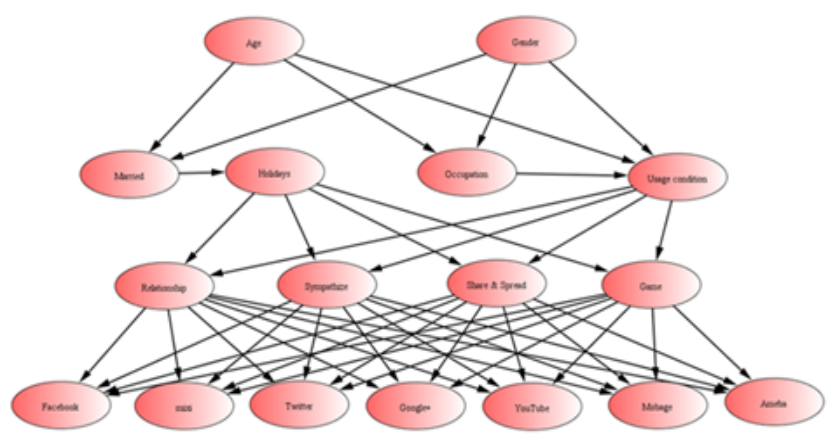

Fig. 2. Built model

TABLE II. NODE AND PARAMETER

\begin{tabular}{|c|c|c|c|c|}
\hline \multirow{2}{*}{ Group name } & $\begin{array}{c}\text { Node in } \\
\text { group }\end{array}$ & \multicolumn{3}{|c|}{ Parameter } \\
\cline { 3 - 5 } & Gender & Male & Female & - \\
\cline { 2 - 5 } Purchaser & Age & $<30$ & $<50$ & $>50$ \\
\cline { 2 - 5 } & Occupation & Student & $\begin{array}{c}\text { Company } \\
\text { employee }\end{array}$ & $\begin{array}{c}\text { School } \\
\text { teacher/staff }\end{array}$ \\
\cline { 2 - 5 } & Married & Married & Single & - \\
\hline $\begin{array}{c}\text { Usage } \\
\text { condition }\end{array}$ & $\begin{array}{c}\text { Usage } \\
\text { condition }\end{array}$ & $\begin{array}{c}\text { More than } \\
5 \text { times a } \\
\text { day }\end{array}$ & $\begin{array}{c}\text { More than } \\
1 \text { times a } \\
\text { day }\end{array}$ & $\begin{array}{c}\text { More than 1 } \\
\text { times a } \\
\text { week }\end{array}$ \\
\hline \multirow{2}{*}{ Usage } & $\begin{array}{c}\text { Relationship, } \\
\text { sympathize, } \\
\text { share \& } \\
\text { spread, game }\end{array}$ & Important & $\begin{array}{c}\text { Ordinary } \\
\text { level }\end{array}$ & $\begin{array}{c}\text { Not } \\
\text { important }\end{array}$ \\
\hline
\end{tabular}

Identify applicable sponsor/s here. If no sponsors, delete this text box (sponsors). 


\begin{tabular}{|c|c|c|c|c|}
\hline Extroversion & Holidays & Outdoor & Indoor & $\begin{array}{c}\text { Cannot } \\
\text { choose } \\
\text { either }\end{array}$ \\
\hline \multirow{3}{*}{ SNS } & $\begin{array}{c}\text { Facebook, } \\
\text { mixi, Twitter, } \\
\text { Google+, } \\
\text { YouTube, } \\
\text { Mobage, } \\
\text { Ameba }\end{array}$ & Use & $\begin{array}{c}\text { Do not } \\
\text { use }\end{array}$ & - \\
& & & \\
\hline
\end{tabular}

\begin{tabular}{|c|c|c|c|c|}
\hline \multirow{2}{*}{$\begin{array}{l}\text { Group } \\
\text { name }\end{array}$} & \multirow{2}{*}{ Node in group } & \multicolumn{3}{|c|}{ Parameter } \\
\hline & & 4 & 5 & 6 \\
\hline \multirow{4}{*}{ Purchaser } & Gender & - & - & - \\
\hline & Age & - & - & - \\
\hline & Occupation & Independents & Part-timers & Others \\
\hline & Married & - & - & - \\
\hline $\begin{array}{c}\text { Usage } \\
\text { condition }\end{array}$ & $\begin{array}{c}\text { Usage } \\
\text { condition }\end{array}$ & $\begin{array}{c}\text { Less than } \\
\text { that }\end{array}$ & - & - \\
\hline $\begin{array}{c}\text { Object of } \\
\text { Usage }\end{array}$ & $\begin{array}{c}\text { Relationship, } \\
\text { sympathize, } \\
\text { share \& spread, } \\
\text { game }\end{array}$ & - & - & - \\
\hline Extroversion & Holidays & - & - & - \\
\hline SNS & $\begin{array}{c}\text { Facebook, } \\
\text { mixi, Twitter, } \\
\text { Google+, } \\
\text { YouTube, } \\
\text { Mobage, } \\
\text { Ameba }\end{array}$ & - & - & - \\
\hline
\end{tabular}

We used BAYONET software (http://www.msi.co.jp/BAYONET/). When plural nodes exist in the same group, the causal relationship is hard to set a priori. In that case, the BAYONET system sets the sequence automatically utilizing AIC standard. Node and parameter of Figure 2 are exhibited in Table 2.

\section{Sensitivity Analysis}

Now, posterior probability is calculated by setting evidence as, for example, 1.0. Comparing Prior probability and Posterior probability, we can seek the change and confirm the instruction for purchasing. We set evidence to all parameters. Therefore the analysis volume becomes too large.

In this paper, we pick up half of the total cases and make analysis. In this paper, detailed analysis is conducted for the objective of usage items, Extroversion items and SNS site items, where nodes we analyze here are "Relationship", "Sympathize", "Share \& Spread", "Game", "Holidays" and "SNS site". The first half part is analyzed in the previous paper.

As stated above, we set evidence for each parameter, and the calculated posterior probability is exhibited in Appendix Table A. The value of "Posterior probability - Prior probability" (we call this "Difference of probability" hereafter) is exhibited in Appendix Table B. The sensitivity analysis is executed by mainly using this table. It is well known that difference of probability becomes small as the node becomes distant [9]. [9].

Here, we pick up major parameters by the distance of node
- Node separated by 1 class: Select major parameter of which absolute value of difference of probability is more than 0.02

- Node separated by 2 class: Select major parameter of which absolute value of difference of probability is more than 0.005

- Node separated by 3 class: Select major parameter of which absolute value of difference of probability is more than 0.001

For the sensitivity analysis of SNS site, only "Use" is analyzed.

In order to assist the definite decision making for the marketing plan, "Less than that" in "Usage condition", "Ordinary" in "Objective of usage" and "Cannot choose either" in "Extroversion" are deleted from the selection.

The larger one for the change of value is selected in "Objective of usage" by comparing "Important" and "Not". Furthermore, when the volume of change in "Not use" of SNS is greater than those in "Use", it is not selected.

In selecting parameters, negative value does not necessarily have distinct meaning, therefore we mainly pick up positive value in the case meaning is not clear.

Now we examine each case.

\section{A. Sensitively Analysis for "Relationship"}

1) Setting evidence to "Important"

(1) Node separated by 1 class.

\begin{tabular}{l|l|l|}
\hline Usage condition & More than 5 times a day & 0.055 \\
\hline
\end{tabular} \begin{tabular}{|l|l|}
\hline (2) Node separated by 2 class & 0.007 \\
\hline
\end{tabular}

(3)Node separated by 3 class

No corresponding data.

We can observe that "Those who put importance concerning, relationship are students who use more than 5 times a day".

2) Setting evidence to "Not important"

(1) Node separated by 1 class

\begin{tabular}{|l|l|l|}
\hline Usage condition & More than 1 times a week & 0.094 \\
\hline Extroversion & Indoor & 0.029 \\
\hline Google+ & Use & 0.102 \\
\hline Mobage & Use & 0.109 \\
\hline Ameba & Use & 0.086 \\
\hline
\end{tabular}

(2)Node separated by 2 class

\begin{tabular}{|l|l|l|}
\hline \multirow{2}{*}{ Gender } & Male & 0.020 \\
\cline { 2 - 3 } & Female & 0.023 \\
\hline \multirow{2}{*}{ Age } & -50 & 0.027 \\
\cline { 2 - 3 } & $50-$ & 0.042 \\
\hline
\end{tabular}




\begin{tabular}{|l|l|l|}
\hline \multirow{4}{*}{ Occupation } & Company Employee & 0.019 \\
\cline { 2 - 3 } & School Teacher/Staff & 0.036 \\
\cline { 2 - 3 } & Independents & 0.019 \\
\cline { 2 - 3 } & Part-timers & 0.037 \\
\hline \multirow{2}{*}{ Married } & Married & 0.027 \\
\cline { 2 - 3 } & Single & 0.016 \\
\hline Sympathize & Not & 0.025 \\
\hline Share \& Spread & Not & 0.046 \\
\hline Game & Not & 0.019 \\
\hline
\end{tabular}

(3) Node separated by 3 class

No corresponding data.

We can observe that "Those who do not put importance concerning relationship, are indoor typed Company Employer, School Teacher/Staff, Independents, Part-timers of married under 50 or over 50 or single Male/Female who use more than 1 times a week, do not esteem Sympathize, Share \& Spread, Game, use Google+, Mobage and Ameba".

B. Sensitivity Analysis for "Sympathize"

1) Setting Evidence to "Important"

(1) Node separated by 1 class

\begin{tabular}{|l|l|l|}
\hline Usage condition & More than 5 times a day & 0.042 \\
\hline Extroversion & Indoor & 0.051 \\
\hline Google+ & Use & 0.046 \\
\hline Ameba & Use & 0.022 \\
\hline
\end{tabular}

(2) Node separated by 2 class

\begin{tabular}{|l|l|l|}
\hline Occupation & Student & 0.011 \\
\hline
\end{tabular}

(3)Node separated by 3 class

No corresponding data.

We can observe that "Those who put importance concerning sympathize, are indoor typed students who use more than 5 times a day, use Google+ and Ameba".

2) Setting evidence to "Not important"

(1) Node separated by 1 class

\begin{tabular}{|l|l|l|}
\hline Usage condition & More than 1 times a week & 0.026 \\
\hline Extroversion & Outdoor & 0.062 \\
\hline Facebook & Use & 0.036 \\
\hline
\end{tabular}

(2)Node separated by 2 class

\begin{tabular}{|l|l|l|}
\hline \multirow{3}{*}{ Gender } & Male & 0.006 \\
\cline { 2 - 3 } & Female & 0.005 \\
\hline \multirow{3}{*}{ Occupation } & -50 & 0.012 \\
\cline { 2 - 3 } & $50-$ & 0.021 \\
\hline & Company Employee & 0.006 \\
\cline { 2 - 3 } & School Teacher/Staff & 0.016 \\
\cline { 2 - 3 } & Independents & 0.008 \\
\cline { 2 - 3 } & Part-timers & 0.016 \\
\hline \multirow{2}{*}{ Married } & Married & 0.017 \\
\hline Relationship & Not & 0.015 \\
\hline
\end{tabular}

\begin{tabular}{|l|l|l|}
\hline Share \& Spread & Not & 0.018 \\
\hline Game & Not & 0.008 \\
\hline
\end{tabular}

(3)Node separated by 3 class

No corresponding data

We can observe that "Those who do not put importance concerning sympathize, are outdoor typed married Male/Female of Company Employee, School Teacher/Staff, Independents, Part-timers under 50 or over 50, do not esteem Relationship, Share \& Spread, nor Game, use Facebook".

C. Sensitivity Analysis for "Share \& Spread"

1) Setting evidence to "Important"

(1) Node separated by 1 class

\begin{tabular}{|l|l|l|}
\hline Usage condition & More than 5 times a day & 0.072 \\
\hline \begin{tabular}{|l|l|} 
(2) Node separated by 2 class & 0.013 \\
\hline Occupation & Student
\end{tabular}
\end{tabular}

\section{(3) Node separated by 3 class}

No corresponding data.

We can observe that "Those who put importance concerning share \& spread are students who use more than 5 times a day".

2) Setting evidence to "Not important"

(1) Node separated by 1 class

\begin{tabular}{|l|l|l|}
\hline Usage condition & More than 1 times a week & 0.064 \\
\hline \multirow{2}{*}{ Extroversion } & Outdoor & 0.026 \\
\cline { 2 - 3 } & Indoor & 0.026 \\
\hline Google+ & Use & 0.124 \\
\hline Mobage & Use & 0.128 \\
\hline Ameba & Use & 0.096 \\
\hline
\end{tabular}

(2)Node separated by 2 class

\begin{tabular}{|l|l|l|}
\hline \multirow{2}{*}{ Gender } & Male & 0.022 \\
\cline { 2 - 3 } & Female & 0.023 \\
\hline \multirow{2}{*}{ Age } & -50 & 0.029 \\
\cline { 2 - 3 } & $50-$ & 0.056 \\
\hline \multirow{3}{*}{ Occupation } & Company Employee & 0.020 \\
\cline { 2 - 3 } & School Teacher/Staff & 0.053 \\
\cline { 2 - 3 } & Independents & 0.020 \\
\cline { 2 - 3 } & Part-timers & 0.038 \\
\hline \multirow{2}{*}{ Married } & Married & 0.032 \\
\hline Relationship & Not & 0.062 \\
\hline Sympathize & Not & 0.030 \\
\hline Game & Not & 0.021 \\
\hline
\end{tabular}

(3) Node separated by 3 class

No corresponding data.

We can observe that "Those who do not put importance concerning share \& spread, are Outdoor/Indoor typed Company Employee, School Teacher/Staff, Independents, Parttimers of married Male/Female under 50 or over 50, do not esteem Relationship, Sympathize, nor Game, use Google+, Mobage and Ameba". 


\section{Sensitivity Analysis for "Game"}

1) Setting evidence to "Important"

(1) Node separated by 1 class

\begin{tabular}{|l|l|l|}
\hline Usage condition & More than 5 times a day & 0.085 \\
\hline Extroversion & Indoor & 0.078 \\
\hline Mobage & Use & 0.150 \\
\hline
\end{tabular}

(2) Node separated by 2 class

\begin{tabular}{|l|l|l|}
\hline Age & -30 & 0.014 \\
\hline Occupation & Student & 0.026 \\
\hline
\end{tabular}

(3) Node separated by 3 class

No corresponding data.

We can observe that "Those who put importance concerning Game, are indoor typed students under 30 who use more than 5 times a day".

2) Setting evidence to "Not important"

(1) Node separated by 1 class

\begin{tabular}{|l|l|l|}
\hline Usage condition & More than 1 times a day & 0.042 \\
\hline Extroversion & Outdoor & 0.027 \\
\hline Facebook & Use & 0.052 \\
\hline
\end{tabular}

(3) Node separated by 3 class

No corresponding data

We can observe that "Those who do not put importance concerning Game, are outdoor typed Company Employee who use more than 1 times a day, use Facebook".

E. Sensitivity Analysis for "Extroversion"

1) Setting evidence to "Outdoor"

(1)Node separated by 1 class

\begin{tabular}{|l|l|l|}
\hline Married & Married & 0.074 \\
\hline Sympathize & Not & 0.054 \\
\hline Game & Not & 0.022 \\
\hline
\end{tabular}

(2)Node separated by 2 class

\begin{tabular}{|l|l|l|}
\hline \multirow{2}{*}{ Gender } & Male & 0.005 \\
\hline \multirow{2}{*}{ Age } & -50 & 0.031 \\
\cline { 2 - 3 } & $50-$ & 0.040 \\
\hline
\end{tabular}

(3) Node separated by 3 class

\begin{tabular}{|l|l|l|}
\hline Occupation & Independents & 0.027 \\
\hline Usage condition & More than 1 times a week & 0.011 \\
\hline
\end{tabular}

We can observe that "Those who prefer outdoor concerning extroversion, are Independent Male over 50 or under 50 who use more than 1 times a week, esteem Sympathize and Game".

2) Setting evidence to "Indoor"

(1) Node separated by 1 class

\begin{tabular}{|l|l|l|}
\hline Married & Single & 0.060 \\
\hline Sympathize & Important & 0.051 \\
\hline Game & Important & 0.095 \\
\hline
\end{tabular}

(2)Node separated by 2 class

\begin{tabular}{|l|l|l|}
\hline Gender & Female & 0.010 \\
\hline Age & -30 & 0.041 \\
\hline Google+ & Use & 0.008 \\
\hline You tube & Use & 0.006 \\
\hline Mobage & Use & 0.026 \\
\hline Ameba & Use & 0.008 \\
\hline
\end{tabular}

(3) Node separated by 3 class

\begin{tabular}{|l|l|l|}
\hline Occupation & Student & 0.040 \\
\hline Usage condition & More than 5 times a day & 0.016 \\
\hline
\end{tabular}

We can observe that "Those who prefer indoor concerning extroversion, are single students under 30 who use more than 5 times a day, esteem Sympathize and Game, use Google+, You tube, Mobage and Ameba".

\section{F. Sensitivity Analysis for "Facebook"}

1) Setting evidence to "Use"

(1) Node separated by 1 class

\begin{tabular}{|l|l|l|}
\hline Relationship & Important & 0.026 \\
\hline Sympathize & Not & 0.045 \\
\hline Share \& Spread & Important & 0.031 \\
\hline Game & Not & 0.065 \\
\hline
\end{tabular}

(2) Node separated by 2 class

\begin{tabular}{|l|l|l|}
\hline Usage condition & More than 1 times a day & 0.006 \\
\hline
\end{tabular}

(3)Node separated by 3 class

No corresponding data.

We can observe that "Those who use Facebook, use more than 1 times a day, esteem Relationship, and Share \& Spread, while do not esteem Sympathize nor Game".

G. Sensitivity Analysis for "mixi"

1) Setting evidence to "Use"

(1) Node separated by 1 class

\begin{tabular}{|l|l|l|}
\hline Relationship & Important & 0.063 \\
\hline Sympathize & Important & 0.033 \\
\hline Share \& Spread & Important & 0.023 \\
\hline Game & Important & 0.058 \\
\hline \multirow{2}{*}{ Extroversion } & Outdoor & 0.032 \\
\cline { 2 - 3 } & Indoor & 0.032 \\
\hline
\end{tabular}

(2) Node separated by 2 class

\begin{tabular}{|l|l|l|}
\hline \multirow{3}{*}{ Usage condition } & More than 5 times a day & 0.048 \\
\cline { 2 - 3 } & More than 1 times a day & 0.037 \\
\cline { 2 - 3 } & More than 1 times a week & 0.016 \\
\hline Facebook & Use & 0.036 \\
\hline Twitter & Use & 0.037 \\
\hline You tube & Use & 0.033 \\
\hline
\end{tabular}

(3)Node separated by 3 class

\begin{tabular}{|l|l|l|}
\hline \multirow{2}{*}{ Gender } & Male & 0.033 \\
\cline { 2 - 3 } & Female & 0.032 \\
\hline Age & -30 & 0.038 \\
\hline
\end{tabular}




\begin{tabular}{|l|l|l|}
\hline \multirow{4}{*}{ Occupation } & -50 & 0.031 \\
\cline { 2 - 3 } & $50-$ & 0.027 \\
\hline & Student & 0.040 \\
\cline { 2 - 3 } & Company Employee & 0.033 \\
\cline { 2 - 3 } & School Teacher/Staff & 0.028 \\
\cline { 2 - 3 } & Independents & 0.033 \\
\cline { 2 - 3 } & Part-timers & 0.028 \\
\hline \multirow{2}{*}{ Married } & Married & 0.031 \\
\cline { 2 - 3 } & Single & 0.034 \\
\hline
\end{tabular}

We can observe that "Those who use mixi, are indoor/outdoor typed Married/Single with versatile occupations in the wide spread ages, esteem Relationship, Sympathize, Share \& Spread and Game, use Facebook, Twitter and You tube".

\section{H. Sensitivity Analysis for "Twitter"}

1) Setting evidence to "Use"

(1) Node separated by 1 class

\begin{tabular}{|l|l|l|}
\hline Relationship & Important & 0.046 \\
\hline Sympathize & Important & 0.063 \\
\hline Share \& Spread & Important & 0.021 \\
\hline Game & Not & 0.048 \\
\hline \multirow{2}{*}{ Extroversion } & Outdoor & 0.030 \\
\cline { 2 - 3 } & Indoor & 0.028 \\
\hline
\end{tabular}

(2) Node separated by 2 class

\begin{tabular}{|l|l|l|}
\hline Usage condition & More than 5 times a day & 0.038 \\
\hline & More than 1 times a day & 0.035 \\
\hline Facebook & More than 1 times a week & 0.022 \\
\hline mixi & Use & 0.034 \\
\hline You tube & Use & 0.034 \\
\hline
\end{tabular}

(3) Node separated by 3 class

\begin{tabular}{|l|l|l|}
\hline \multirow{3}{*}{ Gender } & Male & 0.031 \\
\cline { 2 - 3 } & Female & 0.030 \\
\hline \multirow{4}{*}{ Age } & -30 & 0.033 \\
\cline { 2 - 3 } & -50 & 0.030 \\
\cline { 2 - 3 } & $50-$ & 0.027 \\
\hline \multirow{5}{*}{ Occupation } & Student & 0.034 \\
\cline { 2 - 3 } & Company Employee & 0.031 \\
\cline { 2 - 3 } & School Teacher/Staff & 0.027 \\
\cline { 2 - 3 } & Independents & 0.031 \\
\cline { 2 - 3 } & Part-timers & 0.028 \\
\hline \multirow{3}{*}{ Married } & Married & 0.031 \\
\cline { 2 - 3 } & Single & 0.034 \\
\hline
\end{tabular}

We can observe that "Those who use Twitter, are indoor/outdoor typed Married/Single with versatile occupations in the wide spread ages, esteem Relationship, Sympathize and
Share \& Spread, while do not esteem Game, use Facebook, mixi and You tube".

I. Sensitivity Analysis for "Google+"

1) Setting evidence to "Use"

(1) Node separated by 1 class

\begin{tabular}{|l|l|l|}
\hline Relationship & Not & 0.262 \\
\hline Sympathize & Important & 0.122 \\
\hline Share \& Spread & Not & 0.213 \\
\hline \multirow{2}{*}{ Game } & Important & 0.084 \\
\hline \multirow{2}{*}{ Extroversion } & Outdoor & 0.075 \\
\cline { 2 - 3 } & Indoor & 0.090 \\
\hline
\end{tabular}

(2)Node separated by 2 class

\begin{tabular}{|l|l|l|}
\hline \multirow{3}{*}{ Usage condition } & More than 5 times a day & 0.053 \\
\cline { 2 - 3 } & More than 1 times a day & 0.069 \\
\cline { 2 - 3 } & More than 1 times a week & 0.105 \\
\hline Facebook & Use & 0.063 \\
\hline You tube & Use & 0.082 \\
\hline Mobage & Use & 0.133 \\
\hline Ameba & Use & 0.113 \\
\hline
\end{tabular}

(3) Node separated by 3 class

\begin{tabular}{|l|l|l|}
\hline \multirow{3}{*}{ Gender } & Male & 0.080 \\
\cline { 2 - 3 } & Female & 0.081 \\
\hline \multirow{4}{*}{ Age } & -30 & 0.071 \\
\cline { 2 - 3 } & -50 & 0.083 \\
\cline { 2 - 3 } & $50-$ & 0.093 \\
\hline \multirow{4}{*}{ Occupation } & Student & 0.068 \\
\cline { 2 - 3 } & Company Employee & 0.079 \\
\cline { 2 - 3 } & School Teacher/Staff & 0.091 \\
\cline { 2 - 3 } & Independents & 0.080 \\
\cline { 2 - 3 } & Part-timers & 0.088 \\
\hline \multirow{3}{*}{ Married } & Married & 0.083 \\
\cline { 2 - 3 } & Single & 0.078 \\
\hline
\end{tabular}

We can observe that "Those who use Google+, are indoor/outdoor typed Married/Single with versatile occupations in the wide spread ages, esteem Sympathize and Game, while do not esteem Relationship nor Share \& Spread, use Facebook, mixi and You tube".

\section{J. Sensitivity Analysis for "You tube"}

1) Setting evidence to "Use"

(1)Node separated by 1 class

\begin{tabular}{|l|l|l|}
\hline Sympathize & Important & 0.034 \\
\hline Game & Important & 0.021 \\
\hline
\end{tabular}

(2) Node separated by 2 class

No corresponding data.

(3)Node separated by 3 class

No corresponding data.

We can observe that "Those who use You tube, esteem Sympathize and Game". 
K. Sensitivity Analysis for "Mobage"

1) Setting evidence to "Use"

(1) Node separated by 1 class

\begin{tabular}{|l|l|l|}
\hline Relationship & Not & 0.260 \\
\hline Sympathize & Important & 0.097 \\
\hline Share \& Spread & Not & 0.210 \\
\hline \multirow{2}{*}{ Game } & Important & 0.216 \\
\hline \multirow{2}{*}{ Extroversion } & Outdoor & 0.076 \\
\cline { 2 - 3 } & Indoor & 0.104 \\
\hline
\end{tabular}

(2)Node separated by 2 class

\begin{tabular}{|l|l|l|}
\hline \multirow{3}{*}{ Usage condition } & More than 5 times a day & 0.070 \\
\cline { 2 - 3 } & More than 1 times a day & 0.063 \\
\cline { 2 - 3 } & More than 1 times a week & 0.105 \\
\hline Google+ & Use & 0.128 \\
\hline You tube & Use & 0.086 \\
\hline Ameba & Use & 0.119 \\
\hline
\end{tabular}

(3)Node separated by 3 class

\begin{tabular}{|l|l|l|}
\hline \multirow{3}{*}{ Gender } & Male & 0.083 \\
\cline { 2 - 3 } & Female & 0.083 \\
\hline \multirow{4}{*}{ Age } & -30 & 0.076 \\
\cline { 2 - 3 } & -50 & 0.084 \\
\cline { 2 - 3 } & $50-$ & 0.094 \\
\hline \multirow{4}{*}{ Occupation } & Student & 0.075 \\
\cline { 2 - 3 } & Company Employee & 0.080 \\
\cline { 2 - 3 } & School Teacher/Staff & 0.093 \\
\cline { 2 - 3 } & Independents & 0.082 \\
\cline { 2 - 3 } & Part-timers & 0.089 \\
\hline \multirow{3}{*}{ Married } & Married & 0.084 \\
\cline { 2 - 3 } & Single & 0.083 \\
\hline
\end{tabular}

We can observe that "Those who use Mobage, are indoor/outdoor typed Married/Single with versatile occupations in the wide spread ages, esteem Sympathize and Game, while do not esteem Relationship, nor Share \& Spread, use Google+, You tube and Ameba".

\section{Sensitivity Analysis for "Ameba"}

1) Setting evidence to "Use"

(1) Node separated by 1 class

\begin{tabular}{|l|l|l|}
\hline Relationship & Not & 0.244 \\
\hline Sympathize & Important & 0.113 \\
\hline Share \& Spread & Not & 0.192 \\
\hline \multirow{2}{*}{ Game } & Important & 0.112 \\
\hline \multirow{2}{*}{ Extroversion } & Outdoor & 0.085 \\
\cline { 2 - 3 } & Indoor & 0.095 \\
\hline
\end{tabular}

\section{(2) Node separated by 2 class}

\begin{tabular}{|l|l|l|}
\hline \multirow{3}{*}{ Usage condition } & More than 5 times a day & 0.070 \\
\cline { 2 - 3 } & More than 1 times a day & 0.076 \\
\cline { 2 - 3 } & More than 1 times a week & 0.107 \\
\hline
\end{tabular}

\begin{tabular}{|l|l|l|}
\hline Google + & Use & 0.122 \\
\hline You tube & Use & 0.089 \\
\hline Mobage & Use & 0.130 \\
\hline
\end{tabular}

(3) Node separated by 3 class

\begin{tabular}{|l|l|l|}
\hline \multirow{3}{*}{ Gender } & Male & 0.087 \\
\cline { 2 - 3 } & Female & 0.088 \\
\hline \multirow{4}{*}{ Age } & -30 & 0.081 \\
\cline { 2 - 3 } & -50 & 0.089 \\
\cline { 2 - 3 } & $50-$ & 0.097 \\
\hline \multirow{4}{*}{ Occupation } & Student & 0.079 \\
\cline { 2 - 3 } & Company Employee & 0.086 \\
\cline { 2 - 3 } & School Teacher/Staff & 0.095 \\
\cline { 2 - 3 } & Independents & 0.087 \\
\cline { 2 - 3 } & Part-timers & 0.093 \\
\hline \multirow{3}{*}{ Married } & Married & 0.089 \\
\cline { 2 - 3 } & Single & 0.086 \\
\hline
\end{tabular}

We can observe that "Those who use Ameba, are indoor/outdoor typed Married/Single with versatile occupations in the wide spread ages, esteem Sympathize and Game, while do not esteem Relationship nor Share \& Spread, use Google+, You tube and Mobage".

\section{REMARKS}

Setting evidence to all parameters, we can obtain following findings.

If the model is spread toward lower level with branch, observation data tends to be small. Therefore ripple effect becomes small as it passes through node to node.

The change of differences of probability (ie."Posterior probability - Prior probability") decreases exponentially as a node is separated from the source node where evidence is set. To cope with this, such methods as Reinforcement Learning, transformation by logarithmic scale would be effective. As the depth of a model becomes deep, above phenomenon occurs, therefore model building of shallow depth is required.

1) We could confirm that those who esteem Relationship, Sympathize, Share \& Spread and Game are relatively young and they use them frequently.

2) Those who esteem Sympathize have a tendency to use Google+ and Ameba, while they do not use Facebook. Google+ has a good characteristic in group function and Ameba is often utilized as a diary. Users sympathize these sites rather than Facebook, where it makes public the current information.

Furthermore users feel much more sympathy than Facebook.

This may be because mixi has a good diary function.

3) These who do not esteem Game of outdoor typed company employee often use Facebook.

4) Young people of indoor type use SNS frequently than those of outdoor type. 
5) Correlation between usage objective and SNS site is shown in Table 3.

We can observe that the users of Facebook, mixi, Twitter esteem Relationship and Share \& Spread.

The users of Facebook and Twitter do not esteem Game.

As could be seem from the investigation results of the former half, users of Google+, Mobage, Ameba have the same usage objective and use them simultaneously.

TABLE III. CORRELATION BETWEEN USAGE OBJECTIVE AND SNS SITE

\begin{tabular}{|c|c|c|c|c|}
\hline & Relationship & Sympathize & $\begin{array}{c}\text { Share \& } \\
\text { Spread }\end{array}$ & Game \\
\hline Facebook & $\circ$ & $\times$ & $\circ$ & $\times$ \\
\hline mixi & $\circ$ & $\circ$ & $\circ$ & $\circ$ \\
\hline Twitter & $\circ$ & $\circ$ & $\circ$ & $\times$ \\
\hline Google+ & $\times$ & $\circ$ & $\times$ & $\circ$ \\
\hline You Tube & - & $\circ$ & - & $\circ$ \\
\hline Mobage & $\times$ & $\circ$ & $\times$ & $\circ$ \\
\hline Ameba & $\times$ & $\circ$ & $\times$ & $\circ$ \\
\hline
\end{tabular}

We could obtain the interesting insight by classifying the volume of change.

Further investigation should be executed hereafter.

\section{CONCLUSION}

Social Networking Service (SNS) is prevailing rapidly in Japan in recent years. In this work, a questionnaire investigation was carried out in order to clarify the current usage condition, issues and desired functions. More than 1,000 samples are gathered. Bayesian network is utilized for this analysis. After conducting the sensitivity analysis, useful results are obtained. Differences in usage objectives and SNS sites were made clear by the attributes and preference of SNS users. We could observe that the users of Facebook, mixi,
Twitter esteemed Relationship and Share \& Spread. While the users of Facebook and Twitter did not esteem Game.

They can be utilized effectively for marketing by clarifying the target customer through the sensitivity analysis. We could obtain the detailed results together with those of the former half.

\section{FUTURE WORKS}

To confirm instructions and their results would be our next step investigation. Systematic research investigation for SNS is still on the beginning stage. Such research as this should be further developed with the increased case studies.

\section{REFERENCES}

[1] Kotler, P. and Keller, K., Brady, M., Goodman, M., and Hansen. T, Marketing Management (2 ed.), Pearson, Harlow, UK, 2012.

[2] Neapolitan, R. E, Learning Bayesian Networks, Pearson Prentice Hall, Upper Saddle River, NJ, 2004.

[3] Neaplitan, R.E, Learning Bayesian Networks", Artificial Intelligence, Prentice Hall, 2008.

[4] Tsuji. H., Kojima, M., Takahashi, A., Nakano, M., Aoki, S., Inoue, S., Asari, K., and Mimura, E, Preference mining on future home energy consumption, IEEE International Conference on Systems, Man and Cybernetics, Singapore, pp3697-3701, 2008.

[5] Takahashi. A., Aoki, S., Tsuji, H., and Inoue, S, Bayesian network for future home energy consumption, Lecture Notes in Computer Science, 5243, pp372-379.R. Nicole, "Title of paper with only first word capitalized," J. Name Stand. Abbrev., in press, 2008.

[6] Tatsuoka, K., Yoshida, T., and Munemoto, J, Analysis on purchase behavior according to display way of merchandise by Bayesian network, Journal of Architecture and Planning (Transactions of AIJ), 73(633), pp2349-2354, 2008.

[7] Tatsuoka, K., Yoshida, T., and Munemoto, J, Analysis on relationship between layout of display cases ad purchase behavior by Bayesian network, Journal of Architecture and Planning (Transactions of AIJ), 73(634), pp2633-2638, 2008.

[8] Haga, M. and Motomura, Y, Marketing strategy and product development using Bayesian networks, Jinko Chino Gakkai Jinko Chino Kihon Mondai Kenkyukai Shiryo, 2005, pp59-61.

[9] Takeyasu, K and Ishio, C, Questionnaire Investigation on Jewelry / Accessory and its Analysis Utilizing Bayesian Network, Osaka Prefecture University 『Journal of Economics、Business and Law』 Vol.56 No.1, pp 25-40, 2010.

\section{APPENDIX: Table A}

\begin{tabular}{|c|c|c|c|c|c|c|c|c|c|c|c|c|}
\hline \multirow{2}{*}{ Name } & \multirow{2}{*}{ state } & \multirow{2}{*}{ Prior } & \multicolumn{2}{|c|}{ Gender } & \multicolumn{3}{|c|}{ Age } & \multicolumn{5}{|c|}{ Occupation } \\
\hline & & & Male & Female & -30 & -50 & $50-$ & Student & $\begin{array}{l}\text { Company } \\
\text { Employee }\end{array}$ & $\begin{array}{c}\text { School } \\
\text { Teacher/Staff }\end{array}$ & Independents & Part-timers \\
\hline \multirow{2}{*}{ Gender } & Male & 0.592 & 1.000 & 0.000 & 0.592 & 0.592 & 0.592 & 0.606 & 0.694 & 0.513 & 0.545 & 0.079 \\
\hline & Female & 0.408 & 0.000 & 1.000 & 0.408 & 0.408 & 0.408 & 0.394 & 0.306 & 0.487 & 0.455 & 0.921 \\
\hline \multirow{3}{*}{ Age } & -30 & 0.477 & 0.477 & 0.477 & 1.000 & 0.000 & 0.000 & 0.978 & 0.327 & 0.251 & 0.062 & 0.260 \\
\hline & -50 & 0.449 & 0.449 & 0.449 & 0.000 & 1.000 & 0.000 & 0.014 & 0.584 & 0.657 & 0.806 & 0.582 \\
\hline & $50-$ & 0.075 & 0.074 & 0.074 & 0.000 & 0.000 & 1.000 & 0.008 & 0.090 & 0.092 & 0.133 & 0.158 \\
\hline \multirow{6}{*}{ Occupation } & Student & 0.267 & 0.276 & 0.260 & 0.553 & 0.008 & 0.028 & 1.000 & 0.000 & 0.000 & 0.000 & 0.000 \\
\hline & Company Employee & 0.542 & 0.604 & 0.386 & 0.353 & 0.670 & 0.621 & 0.000 & 1.000 & 0.000 & 0.000 & 0.000 \\
\hline & School Teacher/Staff & 0.040 & 0.037 & 0.051 & 0.022 & 0.062 & 0.052 & 0.000 & 0.000 & 1.000 & 0.000 & 0.000 \\
\hline & Independents & 0.042 & 0.043 & 0.052 & 0.006 & 0.084 & 0.083 & 0.000 & 0.000 & 0.000 & 1.000 & 0.000 \\
\hline & Part-timers & 0.049 & 0.008 & 0.133 & 0.032 & 0.076 & 0.125 & 0.000 & 0.000 & 0.000 & 0.000 & 1.000 \\
\hline & Others & 0.061 & 0.032 & 0.118 & 0.034 & 0.099 & 0.091 & 0.000 & 0.000 & 0.000 & 0.000 & 0.000 \\
\hline \multirow{2}{*}{ Married } & Married & 0.424 & 0.464 & 0.351 & 0.133 & 0.667 & 0.734 & 0.146 & 0.512 & 0.528 & 0.635 & 0.467 \\
\hline & Single & 0.576 & 0.536 & 0.649 & 0.867 & 0.333 & 0.266 & 0.854 & 0.488 & 0.472 & 0.365 & 0.533 \\
\hline
\end{tabular}




\begin{tabular}{|c|c|c|c|c|c|c|c|c|c|c|c|c|}
\hline \multirow{4}{*}{$\begin{array}{l}\text { Usage } \\
\text { condition }\end{array}$} & $\begin{array}{c}\text { More than } 5 \text { times a } \\
\text { day }\end{array}$ & 0.368 & 0.315 & 0.292 & 0.464 & 0.242 & 0.167 & 0.548 & 0.253 & 0.212 & 0.332 & 0.186 \\
\hline & $\begin{array}{l}\text { More than } 1 \text { times a } \\
\text { day }\end{array}$ & 0.408 & 0.382 & 0.395 & 0.375 & 0.388 & 0.351 & 0.322 & 0.441 & 0.372 & 0.369 & 0.360 \\
\hline & $\begin{array}{c}\text { More than } 1 \text { times a } \\
\text { week }\end{array}$ & 0.175 & 0.193 & 0.207 & 0.115 & 0.265 & 0.275 & 0.091 & 0.239 & 0.176 & 0.188 & 0.344 \\
\hline & Less than that & 0.049 & 0.110 & 0.107 & 0.046 & 0.105 & 0.207 & 0.040 & 0.067 & 0.240 & 0.111 & 0.111 \\
\hline \multirow{3}{*}{ Relationship } & Important & 0.824 & 0.788 & 0.785 & 0.822 & 0.775 & 0.743 & 0.832 & 0.789 & 0.753 & 0.790 & 0.757 \\
\hline & Ordinary & 0.102 & 0.118 & 0.119 & 0.100 & 0.124 & 0.141 & 0.094 & 0.118 & 0.138 & 0.117 & 0.132 \\
\hline & Not & 0.074 & 0.094 & 0.095 & 0.078 & 0.101 & 0.116 & 0.074 & 0.093 & 0.110 & 0.093 & 0.111 \\
\hline \multirow{3}{*}{ Sympathize } & Important & 0.385 & 0.368 & 0.368 & 0.389 & 0.358 & 0.345 & 0.396 & 0.365 & 0.353 & 0.367 & 0.353 \\
\hline & Ordinary & 0.311 & 0.322 & 0.323 & 0.312 & 0.327 & 0.331 & 0.308 & 0.325 & 0.327 & 0.321 & 0.331 \\
\hline & Not & 0.304 & 0.310 & 0.309 & 0.299 & 0.315 & 0.324 & 0.297 & 0.310 & 0.319 & 0.312 & 0.316 \\
\hline \multirow{3}{*}{$\begin{array}{c}\text { Share \& } \\
\text { Spread }\end{array}$} & Important & 0.614 & 0.582 & 0.580 & 0.616 & 0.573 & 0.538 & 0.627 & 0.584 & 0.541 & 0.584 & 0.561 \\
\hline & Ordinary & 0.255 & 0.265 & 0.266 & 0.257 & 0.267 & 0.274 & 0.254 & 0.265 & 0.275 & 0.264 & 0.270 \\
\hline & Not & 0.131 & 0.153 & 0.154 & 0.127 & 0.160 & 0.187 & 0.119 & 0.151 & 0.184 & 0.151 & 0.170 \\
\hline \multirow{3}{*}{ Game } & Important & 0.254 & 0.245 & 0.243 & 0.268 & 0.234 & 0.227 & 0.279 & 0.235 & 0.235 & 0.246 & 0.229 \\
\hline & Ordinary & 0.292 & 0.305 & 0.307 & 0.287 & 0.311 & 0.326 & 0.280 & 0.306 & 0.324 & 0.304 & 0.318 \\
\hline & Not & 0.454 & 0.449 & 0.450 & 0.445 & 0.455 & 0.447 & 0.441 & 0.459 & 0.441 & 0.450 & 0.453 \\
\hline \multirow{3}{*}{ Extroversion } & Outdoor & 0.311 & 0.316 & 0.301 & 0.274 & 0.342 & 0.350 & 0.275 & 0.322 & 0.324 & 0.338 & 0.316 \\
\hline & Indoor & 0.269 & 0.263 & 0.279 & 0.310 & 0.234 & 0.225 & 0.308 & 0.256 & 0.254 & 0.239 & 0.263 \\
\hline & Cannot choose either & 0.421 & 0.421 & 0.419 & 0.416 & 0.424 & 0.425 & 0.416 & 0.422 & 0.422 & 0.424 & 0.421 \\
\hline \multirow{2}{*}{ Facebook } & Use & 0.634 & 0.619 & 0.619 & 0.625 & 0.619 & 0.610 & 0.626 & 0.622 & 0.610 & 0.620 & 0.614 \\
\hline & Not & 0.367 & 0.381 & 0.381 & 0.375 & 0.381 & 0.390 & 0.374 & 0.378 & 0.390 & 0.380 & 0.386 \\
\hline \multirow{2}{*}{ mixi } & Use & 0.495 & 0.528 & 0.527 & 0.533 & 0.526 & 0.522 & 0.535 & 0.528 & 0.523 & 0.528 & 0.523 \\
\hline & Not & 0.505 & 0.472 & 0.473 & 0.467 & 0.474 & 0.478 & 0.465 & 0.472 & 0.477 & 0.472 & 0.477 \\
\hline \multirow{2}{*}{ Twitter } & Use & 0.422 & 0.452 & 0.452 & 0.455 & 0.451 & 0.449 & 0.456 & 0.453 & 0.449 & 0.453 & 0.450 \\
\hline & Not & 0.578 & 0.548 & 0.548 & 0.545 & 0.549 & 0.551 & 0.544 & 0.547 & 0.551 & 0.547 & 0.550 \\
\hline \multirow{2}{*}{ Google +} & Use & 0.142 & 0.223 & 0.223 & 0.213 & 0.225 & 0.235 & 0.211 & 0.222 & 0.233 & 0.222 & 0.230 \\
\hline & Not & 0.858 & 0.777 & 0.777 & 0.787 & 0.775 & 0.765 & 0.789 & 0.778 & 0.767 & 0.778 & 0.770 \\
\hline \multirow{2}{*}{ You Tube } & Use & 0.562 & 0.542 & 0.542 & 0.546 & 0.540 & 0.538 & 0.547 & 0.541 & 0.539 & 0.542 & 0.539 \\
\hline & Not & 0.438 & 0.458 & 0.458 & 0.454 & 0.460 & 0.462 & 0.453 & 0.459 & 0.461 & 0.458 & 0.461 \\
\hline \multirow{2}{*}{ Mobage } & Use & 0.116 & 0.199 & 0.199 & 0.192 & 0.200 & 0.209 & 0.191 & 0.196 & 0.209 & 0.198 & 0.205 \\
\hline & Not & 0.884 & 0.801 & 0.801 & 0.808 & 0.800 & 0.791 & 0.809 & 0.804 & 0.791 & 0.802 & 0.795 \\
\hline \multirow{2}{*}{ Ameba } & Use & 0.149 & 0.236 & 0.236 & 0.229 & 0.238 & 0.245 & 0.228 & 0.235 & 0.244 & 0.235 & 0.241 \\
\hline & Not & 0.851 & 0.764 & 0.764 & 0.771 & 0.762 & 0.755 & 0.772 & 0.765 & 0.756 & 0.765 & 0.759 \\
\hline
\end{tabular}

\begin{tabular}{|c|c|c|c|c|c|c|c|c|c|c|c|c|c|c|c|c|}
\hline \multicolumn{2}{|c|}{ Married } & \multicolumn{3}{|c|}{ Usage condition } & \multicolumn{3}{|c|}{ Relationship } & \multicolumn{3}{|c|}{ Sympathize } & \multicolumn{3}{|c|}{ Share \& Spread } & \multicolumn{3}{|c|}{ Game } \\
\hline Married & Single & $\begin{array}{c}\text { More } \\
\text { than } 5 \\
\text { times a } \\
\text { day }\end{array}$ & $\begin{array}{c}\text { More } \\
\text { than } 1 \\
\text { times a } \\
\text { day }\end{array}$ & $\begin{array}{c}\text { More } \\
\text { than } 1 \\
\text { times a } \\
\text { week }\end{array}$ & Important & Ordinary & Not & Important & Ordinary & Not & Important & Ordinary & Not & Important & Ordinary & Not \\
\hline 0.657 & 0.545 & 0.609 & 0.589 & 0.573 & 0.593 & 0.586 & 0.584 & 0.592 & 0.591 & 0.5922 & 0.593 & 0.591 & 0.586 & 0.594 & 0.590 & 0.592 \\
\hline 0.343 & 0.455 & 0.391 & 0.411 & 0.427 & 0.407 & 0.414 & 0.416 & 0.408 & 0.409 & 0.4078 & 0.407 & 0.409 & 0.414 & 0.406 & 0.410 & 0.408 \\
\hline 0.152 & 0.709 & 0.677 & 0.470 & 0.305 & 0.493 & 0.425 & 0.415 & 0.499 & 0.466 & 0.462 & 0.497 & 0.467 & 0.421 & 0.512 & 0.456 & 0.472 \\
\hline 0.717 & 0.257 & 0.283 & 0.460 & 0.591 & 0.436 & 0.487 & 0.494 & 0.431 & 0.458 & 0.460 & 0.433 & 0.456 & 0.489 & 0.419 & 0.464 & 0.453 \\
\hline 0.131 & 0.034 & 0.040 & 0.070 & 0.105 & 0.071 & 0.088 & 0.090 & 0.070 & 0.077 & 0.078 & 0.069 & 0.077 & 0.089 & 0.068 & 0.079 & 0.074 \\
\hline 0.094 & 0.394 & 0.430 & 0.225 & 0.146 & 0.277 & 0.245 & 0.241 & 0.283 & 0.262 & 0.263 & 0.279 & 0.265 & 0.245 & 0.297 & 0.259 & 0.262 \\
\hline 0.636 & 0.437 & 0.409 & 0.598 & 0.593 & 0.516 & 0.512 & 0.508 & 0.510 & 0.520 & 0.517 & 0.516 & 0.515 & 0.509 & 0.494 & 0.517 & 0.525 \\
\hline 0.054 & 0.034 & 0.027 & 0.043 & 0.041 & 0.041 & 0.049 & 0.049 & 0.041 & 0.043 & 0.044 & 0.040 & 0.044 & 0.050 & 0.040 & 0.045 & 0.042 \\
\hline 0.071 & 0.029 & 0.029 & 0.038 & 0.067 & 0.044 & 0.055 & 0.058 & 0.044 & 0.048 & 0.048 & 0.044 & 0.048 & 0.056 & 0.044 & 0.049 & 0.046 \\
\hline 0.065 & 0.051 & 0.033 & 0.052 & 0.096 & 0.056 & 0.069 & 0.072 & 0.056 & 0.061 & 0.061 & 0.055 & 0.061 & 0.069 & 0.055 & 0.062 & 0.059 \\
\hline 0.080 & 0.055 & 0.072 & 0.045 & 0.055 & 0.066 & 0.070 & 0.072 & 0.067 & 0.067 & 0.068 & 0.066 & 0.068 & 0.071 & 0.070 & 0.068 & 0.065 \\
\hline 1.000 & 0.000 & 0.310 & 0.420 & 0.509 & 0.410 & 0.442 & 0.446 & 0.399 & 0.423 & 0.433 & 0.407 & 0.422 & 0.447 & 0.390 & 0.429 & 0.424 \\
\hline 0.000 & 1.000 & 0.690 & 0.580 & 0.491 & 0.590 & 0.558 & 0.554 & 0.601 & 0.577 & 0.567 & 0.593 & 0.578 & 0.553 & 0.610 & 0.571 & 0.576 \\
\hline 0.247 & 0.366 & 1.000 & 0.000 & 0.000 & 0.344 & 0.160 & 0.179 & 0.358 & 0.270 & 0.283 & 0.364 & 0.274 & 0.147 & 0.424 & 0.245 & 0.284 \\
\hline 0.383 & 0.376 & 0.000 & 1.000 & 0.000 & 0.398 & 0.342 & 0.236 & 0.375 & 0.384 & 0.368 & 0.383 & 0.378 & 0.347 & 0.298 & 0.379 & 0.416 \\
\hline
\end{tabular}


(IJACSA) International Journal of Advanced Computer Science and Applications,

Vol. 4, No. 10, 2013

\begin{tabular}{|c|c|c|c|c|c|c|c|c|c|c|c|c|c|c|c|c|}
\hline 0.245 & 0.164 & 0.000 & 0.000 & 1.000 & 0.170 & 0.272 & 0.356 & 0.170 & 0.222 & 0.213 & 0.183 & 0.207 & 0.254 & 0.165 & 0.223 & 0.204 \\
\hline 0.125 & 0.095 & 0.000 & 0.000 & 0.000 & $\begin{array}{l}0.088 \\
\end{array}$ & 0.226 & 0.229 & 0.097 & 0.124 & 0.136 & 0.071 & 0.141 & 0.252 & 0.113 & 0.154 & 0.096 \\
\hline 0.775 & 0.797 & 0.879 & 0.832 & 0.671 & 1.000 & 0.000 & 0.000 & 0.796 & 0.777 & 0.778 & 0.802 & 0.776 & 0.734 & 0.795 & 0.768 & 0.789 \\
\hline 0.125 & 0.113 & 0.064 & 0.108 & 0.161 & 0.000 & 1.000 & 0.000 & 0.114 & 0.123 & 0.123 & 0.110 & 0.124 & 0.146 & 0.114 & 0.129 & 0.118 \\
\hline 0.101 & 0.090 & 0.057 & 0.060 & 0.168 & 0.000 & 0.000 & 1.000 & 0.091 & 0.099 & 0.099 & 0.088 & 0.100 & 0.120 & 0.091 & 0.103 & 0.093 \\
\hline 0.354 & 0.380 & 0.428 & 0.368 & 0.316 & 0.373 & 0.350 & 0.346 & 1.000 & 0.000 & 0.000 & 0.375 & 0.363 & 0.346 & 0.386 & 0.358 & 0.365 \\
\hline 0.326 & 0.318 & 0.285 & 0.329 & 0.354 & 0.320 & 0.332 & 0.335 & 0.000 & 1.000 & 0.000 & 0.318 & 0.326 & 0.332 & 0.311 & 0.331 & 0.324 \\
\hline 0.320 & 0.302 & 0.287 & 0.303 & 0.330 & 0.307 & 0.319 & 0.319 & 0.000 & 0.000 & 1.000 & 0.306 & 0.311 & 0.321 & 0.302 & 0.312 & 0.311 \\
\hline 0.570 & 0.592 & 0.686 & 0.591 & 0.531 & 0.592 & 0.535 & 0.530 & 0.591 & 0.572 & 0.572 & 1.000 & 0.000 & 0.000 & 0.596 & 0.561 & 0.583 \\
\hline 0.267 & 0.263 & 0.238 & 0.267 & 0.273 & 0.263 & 0.276 & 0.277 & 0.262 & 0.268 & 0.267 & 0.000 & 1.000 & 0.000 & 0.259 & 0.271 & 0.265 \\
\hline 0.163 & 0.146 & 0.076 & 0.142 & 0.195 & 0.145 & 0.189 & 0.193 & 0.147 & 0.160 & 0.161 & 0.000 & 0.000 & 1.000 & 0.145 & 0.168 & 0.152 \\
\hline 0.232 & 0.258 & 0.339 & 0.195 & 0.205 & 0.249 & 0.231 & 0.234 & 0.257 & 0.236 & 0.241 & 0.252 & 0.240 & 0.229 & 1.000 & 0.000 & 0.000 \\
\hline 0.312 & 0.299 & 0.245 & 0.308 & 0.339 & 0.301 & 0.328 & 0.330 & 0.298 & 0.314 & 0.309 & 0.297 & 0.313 & 0.331 & 0.000 & 1.000 & 0.000 \\
\hline 0.456 & 0.443 & 0.417 & 0.496 & 0.455 & 0.451 & 0.441 & 0.435 & 0.445 & 0.449 & 0.450 & 0.451 & 0.447 & 0.440 & 0.000 & 0.000 & 1.000 \\
\hline 0.384 & 0.257 & 0.296 & 0.310 & 0.322 & 0.314 & 0.303 & 0.285 & 0.289 & 0.281 & 0.365 & 0.316 & 0.294 & 0.313 & 0.317 & 0.270 & 0.333 \\
\hline 0.187 & 0.329 & 0.285 & 0.269 & 0.257 & 0.265 & 0.286 & 0.288 & 0.320 & 0.251 & 0.230 & 0.272 & 0.264 & 0.270 & 0.364 & 0.239 & 0.240 \\
\hline 0.429 & 0.414 & 0.419 & 0.420 & 0.422 & 0.421 & 0.410 & 0.427 & 0.391 & 0.468 & 0.405 & 0.412 & 0.441 & 0.416 & 0.319 & 0.491 & 0.427 \\
\hline 0.619 & 0.619 & 0.629 & 0.639 & 0.601 & 0.659 & 0.490 & 0.449 & 0.558 & 0.631 & 0.679 & 0.665 & 0.574 & 0.526 & 0.498 & 0.595 & 0.699 \\
\hline 0.381 & 0.381 & 0.371 & 0.361 & 0.399 & 0.341 & 0.510 & 0.551 & 0.442 & 0.369 & 0.321 & 0.335 & 0.426 & 0.474 & 0.502 & 0.405 & 0.301 \\
\hline 0.526 & $\begin{array}{l}0.529 \\
\end{array}$ & 0.543 & 0.532 & 0.511 & 0.558 & 0.424 & $\begin{array}{l}0.408 \\
\end{array}$ & 0.528 & 0.530 & 0.523 & 0.518 & 0.561 & 0.503 & 0.553 & 0.532 & 0.508 \\
\hline 0.474 & 0.471 & 0.457 & 0.468 & 0.489 & 0.442 & 0.576 & 0.592 & 0.472 & 0.470 & 0.477 & 0.482 & 0.439 & 0.497 & 0.447 & 0.468 & 0.492 \\
\hline 0.451 & 0.453 & 0.460 & 0.457 & 0.444 & 0.468 & 0.373 & 0.425 & 0.485 & 0.427 & 0.440 & 0.443 & 0.491 & 0.422 & 0.423 & 0.450 & 0.470 \\
\hline 0.549 & 0.547 & 0.540 & 0.543 & 0.556 & 0.532 & 0.627 & 0.575 & 0.515 & 0.573 & 0.560 & 0.557 & 0.509 & 0.578 & 0.577 & 0.550 & 0.530 \\
\hline 0.226 & 0.221 & 0.195 & 0.212 & 0.248 & 0.188 & 0.303 & 0.405 & 0.264 & 0.213 & 0.187 & 0.178 & 0.238 & 0.355 & 0.226 & 0.245 & 0.208 \\
\hline 0.774 & 0.779 & 0.805 & 0.788 & 0.752 & 0.812 & 0.697 & 0.595 & 0.736 & 0.787 & 0.813 & 0.822 & 0.762 & 0.645 & 0.774 & 0.755 & 0.792 \\
\hline 0.540 & 0.544 & 0.555 & 0.540 & 0.534 & 0.542 & 0.576 & 0.507 & 0.596 & 0.502 & 0.520 & 0.547 & 0.551 & 0.513 & 0.583 & 0.532 & 0.527 \\
\hline 0.460 & 0.456 & 0.445 & 0.460 & 0.466 & 0.458 & 0.424 & 0.493 & 0.404 & 0.498 & 0.480 & 0.453 & 0.449 & 0.487 & 0.417 & 0.468 & 0.473 \\
\hline 0.200 & 0.199 & 0.186 & 0.179 & 0.221 & 0.159 & 0.322 & 0.376 & 0.213 & 0.236 & 0.145 & 0.166 & 0.196 & 0.326 & 0.332 & 0.201 & 0.127 \\
\hline 0.800 & 0.801 & 0.814 & 0.821 & 0.779 & 0.841 & 0.678 & 0.624 & 0.787 & 0.764 & 0.855 & 0.834 & 0.804 & 0.674 & 0.668 & 0.799 & 0.873 \\
\hline 0.238 & 0.235 & 0.219 & 0.225 & 0.255 & 0.209 & 0.293 & 0.392 & 0.261 & 0.211 & 0.232 & 0.206 & 0.238 & 0.341 & 0.261 & 0.254 & 0.212 \\
\hline 0.762 & 0.765 & 0.781 & 0.775 & 0.745 & 0.791 & 0.707 & 0.608 & 0.739 & 0.789 & 0.768 & 0.794 & 0.762 & 0.659 & 0.739 & 0.746 & 0.788 \\
\hline
\end{tabular}

\begin{tabular}{|c|c|c|c|c|c|c|c|c|c|c|c|c|c|c|c|}
\hline \multicolumn{2}{|c|}{ Extroversion } & \multicolumn{2}{|c|}{ Facebook } & \multicolumn{2}{|c|}{$\operatorname{mixi}$} & \multicolumn{2}{|c|}{ Twitter } & \multicolumn{2}{|c|}{ Google +} & \multicolumn{2}{|c|}{ You Tube } & \multicolumn{2}{|c|}{ Mobage } & \multicolumn{2}{|c|}{ Ameba } \\
\hline Outdoor & Indoor & Use & Not & Use & Not & Use & Not & Use & Not & Use & Not & Use & Not & Use & Not \\
\hline 0.603 & 0.577 & 0.592 & 0.591 & 0.5921 & 0.5915 & 0.592 & 0.5917 & 0.5902 & 0.5922 & 0.5919 & 0.5917 & 0.5901 & 0.5922 & 0.5908 & 0.5921 \\
\hline 0.397 & 0.423 & 0.408 & 0.409 & 0.408 & 0.409 & 0.408 & 0.408 & 0.410 & 0.408 & 0.408 & 0.408 & 0.410 & 0.408 & 0.409 & 0.408 \\
\hline 0.421 & 0.548 & 0.480 & 0.472 & 0.480 & 0.473 & 0.479 & 0.475 & 0.462 & 0.481 & 0.479 & 0.474 & 0.465 & 0.480 & 0.467 & 0.480 \\
\hline 0.495 & 0.390 & 0.447 & 0.452 & 0.446 & 0.452 & 0.447 & 0.450 & 0.459 & 0.446 & 0.447 & 0.451 & 0.457 & 0.447 & 0.456 & 0.446 \\
\hline 0.084 & 0.062 & 0.074 & 0.076 & 0.074 & 0.075 & 0.074 & 0.075 & 0.078 & 0.073 & 0.074 & 0.075 & 0.078 & 0.074 & 0.077 & 0.074 \\
\hline 0.240 & 0.308 & 0.270 & 0.269 & 0.271 & 0.267 & 0.270 & 0.269 & 0.263 & 0.271 & 0.271 & 0.268 & 0.267 & 0.270 & 0.266 & 0.271 \\
\hline 0.535 & 0.490 & 0.517 & 0.511 & 0.515 & 0.515 & 0.516 & 0.515 & 0.513 & 0.516 & 0.514 & 0.516 & 0.509 & 0.517 & 0.513 & 0.516 \\
\hline 0.045 & 0.040 & 0.042 & 0.043 & 0.042 & 0.043 & 0.042 & 0.043 & 0.044 & 0.042 & 0.042 & 0.043 & 0.044 & 0.042 & 0.044 & 0.042 \\
\hline 0.051 & 0.041 & 0.046 & 0.048 & 0.046 & 0.047 & 0.046 & 0.047 & 0.049 & 0.046 & 0.046 & 0.047 & 0.049 & 0.046 & 0.048 & 0.046 \\
\hline 0.060 & 0.057 & 0.058 & 0.060 & 0.058 & 0.059 & 0.058 & 0.059 & 0.062 & 0.058 & 0.058 & 0.059 & 0.062 & 0.058 & 0.061 & 0.058 \\
\hline 0.070 & 0.064 & 0.067 & 0.068 & 0.067 & 0.067 & 0.067 & 0.067 & 0.069 & 0.067 & 0.067 & 0.067 & 0.069 & 0.067 & 0.068 & 0.067 \\
\hline 0.517 & 0.289 & 0.417 & 0.418 & 0.416 & 0.420 & 0.416 & 0.418 & 0.424 & 0.416 & 0.416 & 0.420 & 0.420 & 0.417 & 0.422 & 0.416 \\
\hline 0.483 & 0.711 & 0.583 & 0.582 & 0.584 & 0.580 & 0.584 & 0.582 & 0.576 & 0.584 & 0.584 & 0.580 & 0.580 & 0.583 & 0.578 & 0.584 \\
\hline 0.294 & 0.323 & 0.312 & 0.296 & 0.315 & 0.296 & 0.311 & 0.302 & 0.269 & 0.317 & 0.313 & 0.298 & 0.282 & 0.312 & 0.283 & 0.313 \\
\hline 0.376 & 0.375 & 0.388 & 0.356 & 0.379 & 0.372 & 0.380 & 0.372 & 0.356 & 0.381 & 0.375 & 0.377 & 0.338 & 0.386 & 0.358 & 0.381 \\
\hline 0.208 & 0.189 & 0.194 & 0.209 & 0.193 & 0.207 & 0.196 & 0.203 & 0.222 & 0.194 & 0.197 & 0.204 & 0.221 & 0.195 & 0.215 & 0.195 \\
\hline 0.121 & 0.112 & 0.106 & 0.138 & 0.112 & 0.124 & 0.113 & 0.122 & 0.153 & 0.108 & 0.116 & 0.121 & 0.159 & 0.108 & 0.144 & 0.110 \\
\hline 0.794 & 0.770 & 0.836 & 0.700 & 0.830 & 0.734 & 0.812 & 0.762 & 0.659 & 0.820 & 0.785 & 0.784 & 0.624 & 0.824 & 0.692 & 0.813 \\
\hline 0.116 & 0.128 & 0.095 & 0.160 & 0.097 & 0.145 & 0.098 & 0.137 & 0.165 & 0.106 & 0.126 & 0.112 & 0.193 & 0.101 & 0.148 & 0.111 \\
\hline 0.089 & 0.103 & 0.069 & 0.140 & 0.074 & 0.121 & 0.090 & 0.101 & 0.175 & 0.073 & 0.089 & 0.104 & 0.183 & 0.075 & 0.160 & 0.076 \\
\hline
\end{tabular}


(IJACSA) International Journal of Advanced Computer Science and Applications,

Vol. 4, No. 10, 2013

\begin{tabular}{|c|c|c|c|c|c|c|c|c|c|c|c|c|c|c|c|}
\hline 0.343 & 0.437 & 0.331 & 0.427 & 0.368 & 0.367 & 0.394 & 0.346 & 0.432 & 0.348 & 0.404 & 0.325 & 0.393 & 0.361 & 0.407 & 0.355 \\
\hline 0.291 & 0.299 & 0.329 & 0.312 & 0.325 & 0.320 & 0.304 & 0.338 & 0.308 & 0.327 & 0.298 & 0.351 & 0.381 & 0.308 & 0.289 & 0.333 \\
\hline 0.366 & 0.264 & 0.340 & 0.261 & 0.307 & 0.313 & 0.302 & 0.316 & 0.260 & 0.325 & 0.298 & 0.324 & 0.226 & 0.331 & 0.304 & 0.311 \\
\hline 0.590 & 0.584 & 0.622 & 0.510 & 0.569 & 0.590 & 0.567 & 0.589 & 0.463 & 0.613 & 0.584 & 0.573 & 0.481 & 0.603 & 0.506 & 0.602 \\
\hline 0.252 & 0.259 & 0.246 & 0.297 & 0.283 & 0.246 & 0.289 & 0.247 & 0.282 & 0.261 & 0.270 & 0.261 & 0.260 & 0.267 & 0.266 & 0.265 \\
\hline 0.157 & 0.157 & 0.132 & 0.193 & 0.148 & 0.164 & 0.144 & 0.165 & 0.255 & 0.127 & 0.146 & 0.166 & 0.260 & 0.129 & 0.228 & 0.133 \\
\hline 0.251 & 0.332 & 0.197 & 0.322 & 0.257 & 0.232 & 0.230 & 0.258 & 0.247 & 0.245 & 0.264 & 0.223 & 0.403 & 0.204 & 0.270 & 0.237 \\
\hline 0.268 & 0.270 & 0.296 & 0.324 & 0.311 & 0.302 & 0.305 & 0.308 & 0.336 & 0.298 & 0.301 & 0.314 & 0.309 & 0.306 & 0.328 & 0.300 \\
\hline 0.481 & 0.398 & 0.506 & 0.354 & 0.432 & 0.466 & 0.466 & 0.434 & 0.417 & 0.457 & 0.435 & 0.463 & 0.287 & 0.489 & 0.401 & 0.463 \\
\hline 1.000 & 0.000 & 0.315 & 0.302 & 0.310 & 0.310 & 0.310 & 0.310 & 0.302 & 0.312 & 0.309 & 0.311 & 0.298 & 0.313 & 0.306 & 0.311 \\
\hline 0.000 & 1.000 & 0.259 & 0.286 & 0.270 & 0.269 & 0.269 & 0.270 & 0.277 & 0.267 & 0.275 & 0.264 & 0.295 & 0.263 & 0.277 & 0.267 \\
\hline 0.000 & 0.000 & 0.426 & 0.413 & 0.420 & 0.420 & 0.421 & 0.420 & 0.421 & 0.421 & 0.416 & 0.426 & 0.408 & 0.424 & 0.417 & 0.422 \\
\hline 0.628 & 0.595 & 1.000 & 0.000 & 0.623 & 0.613 & 0.623 & 0.614 & 0.570 & 0.632 & 0.613 & 0.625 & 0.542 & 0.639 & 0.581 & 0.630 \\
\hline 0.372 & 0.405 & 0.000 & 1.000 & 0.377 & 0.387 & 0.377 & 0.386 & 0.430 & 0.368 & 0.387 & 0.375 & 0.458 & 0.361 & 0.419 & 0.370 \\
\hline 0.527 & 0.527 & 0.5311 & 0.5189 & 1.000 & 0.000 & 0.532 & 0.524 & 0.509 & 0.532 & 0.528 & 0.526 & 0.507 & 0.531 & 0.514 & 0.531 \\
\hline 0.473 & 0.473 & 0.4689 & 0.4811 & 0.000 & 1.000 & 0.468 & 0.476 & 0.491 & 0.468 & 0.472 & 0.474 & 0.493 & 0.469 & 0.486 & 0.469 \\
\hline 0.452 & 0.450 & 0.4556 & 0.4454 & 0.456 & 0.448 & 1.000 & 0.000 & 0.446 & 0.454 & 0.453 & 0.451 & 0.433 & 0.457 & 0.446 & 0.454 \\
\hline 0.548 & 0.550 & 0.5444 & 0.5546 & 0.544 & 0.552 & 0.000 & 1.000 & 0.554 & 0.546 & 0.547 & 0.549 & 0.567 & 0.543 & 0.554 & 0.546 \\
\hline 0.217 & 0.232 & 0.2056 & 0.2516 & 0.216 & 0.232 & 0.221 & 0.226 & 1.000 & 0.000 & 0.224 & 0.223 & 0.275 & 0.211 & 0.255 & 0.213 \\
\hline 0.783 & 0.768 & 0.7944 & 0.7484 & 0.784 & 0.768 & 0.779 & 0.774 & 0.000 & 1.000 & 0.776 & 0.777 & 0.725 & 0.789 & 0.745 & 0.787 \\
\hline 0.541 & 0.551 & 0.5369 & 0.5490 & 0.543 & 0.540 & 0.543 & 0.541 & 0.543 & 0.541 & 1.000 & 0.000 & 0.548 & 0.540 & 0.543 & 0.541 \\
\hline 0.459 & 0.449 & 0.4631 & 0.4510 & 0.457 & 0.460 & 0.457 & 0.459 & 0.457 & 0.459 & 0.000 & 1.000 & 0.452 & 0.460 & 0.457 & 0.459 \\
\hline 0.192 & 0.220 & 0.1730 & 0.2416 & 0.194 & 0.207 & 0.191 & 0.207 & 0.244 & 0.187 & 0.202 & 0.197 & 1.000 & 0.000 & 0.234 & 0.189 \\
\hline 0.808 & 0.780 & 0.8270 & 0.7584 & 0.806 & 0.793 & 0.809 & 0.793 & 0.756 & 0.813 & 0.798 & 0.803 & 0.000 & 1.000 & 0.766 & 0.811 \\
\hline 0.234 & 0.244 & 0.2220 & 0.2595 & 0.231 & 0.243 & 0.234 & 0.239 & 0.271 & 0.227 & 0.237 & 0.236 & 0.278 & 0.226 & 1.000 & 0.000 \\
\hline 0.766 & 0.756 & 0.7780 & 0.7405 & 0.769 & 0.757 & 0.766 & 0.761 & 0.729 & 0.773 & 0.763 & 0.764 & 0.722 & 0.774 & 0.000 & 1.000 \\
\hline
\end{tabular}

\section{APPENDIX: Table B}

\begin{tabular}{|c|c|c|c|c|c|c|c|c|c|c|c|c|}
\hline \multirow{2}{*}{ Name } & \multirow{2}{*}{ state } & \multirow{2}{*}{ Prior } & \multicolumn{2}{|c|}{ Gender } & \multicolumn{3}{|c|}{ Age } & \multicolumn{5}{|c|}{ Occupation } \\
\hline & & & Male & Female & -30 & -50 & $50-$ & Student & Company Employee & School Teacher/Staff & Independents & Part-timers \\
\hline \multirow{2}{*}{ Gender } & Male & 0.592 & & & 0.000 & 0.000 & 0.000 & 0.014 & 0.102 & -0.079 & -0.047 & -0.513 \\
\hline & Female & 0.408 & & & 0.000 & 0.000 & 0.000 & -0.014 & -0.102 & 0.079 & 0.047 & 0.513 \\
\hline \multirow{3}{*}{ Age } & -30 & 0.477 & 0.000 & 0.000 & & & & 0.502 & -0.150 & -0.226 & -0.415 & -0.217 \\
\hline & -50 & 0.449 & 0.000 & 0.000 & & & & -0.435 & 0.135 & 0.209 & 0.357 & 0.133 \\
\hline & $50-$ & 0.075 & 0.000 & 0.000 & & & & -0.067 & 0.015 & 0.017 & 0.058 & 0.084 \\
\hline \multirow{6}{*}{ Occupation } & Student & 0.267 & 0.009 & -0.007 & 0.286 & -0.259 & -0.240 & & & & & \\
\hline & Company Employee & 0.542 & 0.063 & -0.156 & -0.189 & 0.129 & 0.079 & & & & & \\
\hline & School Teacher/Staff & 0.040 & -0.003 & 0.011 & -0.017 & 0.022 & 0.012 & & & & & \\
\hline & Independents & 0.042 & 0.001 & 0.010 & -0.036 & 0.042 & 0.041 & & & & & \\
\hline & Part-timers & 0.049 & -0.041 & 0.084 & -0.017 & 0.027 & 0.076 & & & & & \\
\hline & Others & 0.061 & -0.029 & 0.057 & -0.027 & 0.038 & 0.030 & & & & & \\
\hline \multirow{2}{*}{ Married } & Married & 0.424 & 0.040 & -0.073 & -0.290 & 0.243 & 0.310 & -0.277 & 0.089 & 0.104 & 0.211 & 0.044 \\
\hline & Single & 0.576 & -0.040 & 0.073 & 0.290 & -0.243 & -0.310 & 0.277 & -0.089 & -0.104 & -0.211 & -0.044 \\
\hline \multirow{4}{*}{ Usage condition } & More than 5 times a day & 0.368 & -0.053 & -0.076 & 0.096 & -0.126 & -0.201 & 0.180 & -0.115 & -0.156 & -0.036 & -0.182 \\
\hline & More than 1 times a day & 0.408 & -0.026 & -0.014 & -0.033 & -0.020 & -0.058 & -0.086 & 0.033 & -0.036 & -0.039 & -0.049 \\
\hline & More than 1 times a week & 0.175 & 0.018 & 0.032 & -0.059 & 0.091 & 0.100 & -0.084 & 0.064 & 0.001 & 0.014 & 0.169 \\
\hline & Less than that & 0.049 & 0.061 & 0.058 & -0.003 & 0.056 & 0.158 & -0.009 & 0.018 & 0.191 & 0.062 & 0.062 \\
\hline \multirow{3}{*}{ Relationship } & Important & 0.824 & -0.036 & -0.039 & -0.002 & -0.049 & -0.081 & 0.007 & -0.035 & -0.072 & -0.034 & -0.067 \\
\hline & Ordinary & 0.102 & 0.016 & 0.018 & -0.002 & 0.022 & 0.039 & -0.008 & 0.016 & 0.036 & 0.015 & 0.030 \\
\hline & Not & 0.074 & 0.020 & 0.022 & 0.004 & 0.027 & 0.042 & 0.000 & 0.019 & 0.036 & 0.019 & 0.037 \\
\hline \multirow{3}{*}{ Sympathize } & Important & 0.385 & -0.017 & -0.017 & 0.004 & -0.027 & -0.040 & 0.011 & -0.021 & -0.032 & -0.018 & -0.032 \\
\hline & Ordinary & 0.311 & 0.011 & 0.012 & 0.001 & 0.015 & 0.020 & -0.004 & 0.014 & 0.016 & 0.010 & 0.020 \\
\hline & Not & 0.304 & 0.006 & 0.005 & -0.005 & 0.012 & 0.021 & -0.007 & 0.006 & 0.016 & 0.008 & 0.012 \\
\hline \multirow{2}{*}{ Share \& Spread } & Important & 0.614 & -0.032 & -0.034 & 0.002 & -0.041 & -0.076 & 0.013 & -0.030 & -0.073 & -0.030 & -0.053 \\
\hline & Ordinary & 0.255 & 0.010 & 0.011 & 0.002 & 0.012 & 0.020 & -0.001 & 0.010 & 0.020 & 0.010 & 0.015 \\
\hline
\end{tabular}


(IJACSA) International Journal of Advanced Computer Science and Applications,

Vol. 4, No. 10, 2013

\begin{tabular}{|c|c|c|c|c|c|c|c|c|c|c|c|c|}
\hline & Not & 0.131 & 0.022 & 0.023 & -0.004 & 0.029 & 0.056 & -0.012 & 0.020 & 0.053 & 0.020 & 0.038 \\
\hline \multirow{3}{*}{ Game } & Important & 0.254 & -0.008 & -0.010 & 0.014 & -0.020 & -0.027 & 0.026 & -0.019 & -0.018 & -0.008 & -0.025 \\
\hline & Ordinary & 0.292 & 0.013 & 0.015 & -0.005 & 0.019 & 0.034 & -0.012 & 0.014 & 0.031 & 0.012 & 0.026 \\
\hline & Not & 0.454 & -0.005 & -0.004 & -0.009 & 0.001 & -0.007 & -0.013 & 0.005 & -0.013 & -0.004 & -0.001 \\
\hline \multirow{3}{*}{ Extroversion } & Outdoor & 0.311 & 0.005 & -0.009 & -0.037 & 0.031 & 0.040 & -0.035 & 0.011 & 0.013 & 0.027 & 0.006 \\
\hline & Indoor & 0.269 & -0.006 & 0.010 & 0.041 & -0.035 & -0.044 & 0.040 & -0.013 & -0.015 & -0.030 & -0.006 \\
\hline & Cannot choose either & 0.421 & 0.000 & -0.001 & -0.005 & 0.003 & 0.004 & -0.004 & 0.001 & 0.001 & 0.003 & 0.000 \\
\hline \multirow{2}{*}{ Facebook } & Use & 0.634 & -0.014 & -0.015 & -0.009 & -0.015 & -0.024 & -0.008 & -0.011 & -0.024 & -0.013 & -0.019 \\
\hline & Not & 0.367 & 0.014 & 0.015 & 0.009 & 0.015 & 0.024 & 0.008 & 0.011 & 0.024 & 0.013 & 0.019 \\
\hline \multirow{2}{*}{$\operatorname{mixi}$} & Use & 0.495 & 0.033 & 0.032 & 0.038 & 0.031 & 0.027 & 0.040 & 0.033 & 0.028 & 0.033 & 0.028 \\
\hline & Not & 0.505 & -0.033 & -0.032 & -0.038 & -0.031 & -0.027 & -0.040 & -0.033 & -0.028 & -0.033 & -0.028 \\
\hline \multirow{2}{*}{ Twitter } & Use & 0.422 & 0.031 & 0.030 & 0.033 & 0.030 & 0.027 & 0.034 & 0.031 & 0.027 & 0.031 & 0.028 \\
\hline & Not & 0.578 & -0.031 & -0.030 & -0.033 & -0.030 & -0.027 & -0.034 & -0.031 & -0.027 & -0.031 & -0.028 \\
\hline \multirow{2}{*}{ Google+ } & Use & 0.142 & 0.080 & 0.081 & 0.071 & 0.083 & 0.093 & 0.068 & 0.079 & 0.091 & 0.080 & 0.088 \\
\hline & Not & 0.858 & -0.080 & -0.081 & -0.071 & -0.083 & -0.093 & -0.068 & -0.079 & -0.091 & -0.080 & -0.088 \\
\hline \multirow{2}{*}{ You Tube } & Use & 0.562 & -0.020 & -0.020 & -0.016 & -0.022 & -0.024 & -0.015 & -0.021 & -0.022 & -0.020 & -0.023 \\
\hline & Not & 0.438 & 0.020 & 0.020 & 0.016 & 0.022 & 0.024 & 0.015 & 0.021 & 0.022 & 0.020 & 0.023 \\
\hline \multirow{2}{*}{ Mobage } & Use & 0.116 & 0.083 & 0.083 & 0.076 & 0.084 & 0.094 & 0.075 & 0.080 & 0.093 & 0.082 & 0.089 \\
\hline & Not & 0.884 & -0.083 & -0.083 & -0.076 & -0.084 & -0.094 & -0.075 & -0.080 & -0.093 & -0.082 & -0.089 \\
\hline \multirow{2}{*}{ Ameba } & Use & 0.149 & 0.087 & 0.088 & 0.081 & 0.089 & 0.097 & 0.079 & 0.086 & 0.095 & 0.087 & 0.093 \\
\hline & Not & 0.851 & -0.087 & -0.088 & -0.081 & -0.089 & -0.097 & -0.079 & -0.086 & -0.095 & -0.087 & -0.093 \\
\hline
\end{tabular}

\begin{tabular}{|c|c|c|c|c|c|c|c|c|c|c|c|c|c|c|c|c|}
\hline \multicolumn{2}{|c|}{ Married } & \multicolumn{3}{|c|}{ Usage condition } & \multicolumn{3}{|c|}{ Relationship } & \multicolumn{3}{|c|}{ Sympathize } & \multicolumn{3}{|c|}{ Share \& Spread } & \multicolumn{3}{|c|}{ Game } \\
\hline Married & Single & $\begin{array}{c}\text { More } \\
\text { than } 5 \\
\text { times a } \\
\text { day }\end{array}$ & $\begin{array}{c}\text { More } \\
\text { than } 1 \\
\text { times a } \\
\text { day }\end{array}$ & $\begin{array}{c}\text { More } \\
\text { than } 1 \\
\text { times } \\
\text { a week }\end{array}$ & Important & Ordinary & Not & Important & Ordinary & Not & Important & Ordinary & Not & Important & Ordinary & Not \\
\hline 0.065 & -0.047 & 0.017 & -0.003 & -0.019 & 0.001 & -0.006 & -0.008 & 0.001 & -0.001 & 0.000 & 0.002 & -0.001 & -0.006 & 0.002 & -0.002 & 0.000 \\
\hline-0.065 & 0.047 & -0.017 & 0.003 & 0.019 & -0.001 & 0.006 & 0.008 & -0.001 & 0.001 & 0.000 & -0.002 & 0.001 & 0.006 & -0.002 & 0.002 & 0.000 \\
\hline-0.324 & 0.233 & 0.200 & -0.006 & -0.172 & 0.016 & -0.051 & -0.061 & 0.023 & -0.011 & -0.014 & 0.021 & -0.010 & -0.055 & 0.035 & -0.020 & -0.005 \\
\hline 0.268 & -0.192 & -0.165 & 0.011 & 0.142 & -0.012 & 0.038 & 0.046 & -0.018 & 0.009 & 0.011 & -0.015 & 0.007 & 0.040 & -0.029 & 0.016 & 0.005 \\
\hline 0.056 & -0.041 & -0.035 & -0.005 & 0.030 & -0.004 & 0.013 & 0.016 & -0.005 & 0.002 & 0.003 & -0.005 & 0.003 & 0.015 & -0.006 & 0.005 & 0.000 \\
\hline-0.173 & 0.127 & 0.162 & -0.042 & -0.121 & 0.010 & -0.022 & -0.027 & 0.016 & -0.006 & -0.005 & 0.011 & -0.002 & -0.022 & 0.030 & -0.008 & -0.005 \\
\hline 0.095 & -0.105 & -0.133 & 0.056 & 0.052 & -0.026 & -0.029 & -0.034 & -0.032 & -0.022 & -0.025 & -0.025 & -0.027 & -0.033 & -0.048 & -0.025 & -0.016 \\
\hline 0.014 & -0.005 & -0.013 & 0.003 & 0.002 & 0.001 & 0.009 & 0.009 & 0.001 & 0.003 & 0.004 & 0.000 & 0.004 & 0.011 & 0.000 & 0.005 & 0.002 \\
\hline 0.029 & -0.013 & -0.013 & -0.004 & 0.026 & 0.002 & 0.013 & 0.016 & 0.002 & 0.006 & 0.007 & 0.002 & 0.006 & 0.014 & 0.002 & 0.008 & 0.004 \\
\hline 0.016 & 0.002 & -0.015 & 0.003 & 0.047 & 0.007 & 0.020 & 0.024 & 0.007 & 0.012 & 0.012 & 0.006 & 0.012 & 0.021 & 0.006 & 0.013 & 0.010 \\
\hline 0.019 & -0.006 & 0.011 & -0.016 & -0.006 & 0.005 & 0.009 & 0.011 & 0.006 & 0.006 & 0.007 & 0.005 & 0.007 & 0.010 & 0.009 & 0.007 & 0.004 \\
\hline & & -0.114 & -0.003 & 0.085 & -0.014 & 0.019 & 0.023 & -0.024 & 0.000 & 0.009 & -0.017 & -0.001 & 0.023 & -0.033 & 0.005 & 0.001 \\
\hline & & 0.114 & 0.003 & -0.085 & 0.014 & -0.019 & -0.023 & 0.024 & 0.000 & -0.009 & 0.017 & 0.001 & -0.023 & 0.033 & -0.005 & -0.001 \\
\hline-0.121 & -0.002 & & & & -0.024 & -0.208 & -0.189 & -0.010 & -0.098 & -0.085 & -0.004 & -0.094 & -0.221 & 0.056 & -0.123 & -0.083 \\
\hline-0.025 & -0.033 & & & & -0.010 & -0.066 & -0.172 & -0.033 & -0.024 & -0.041 & -0.026 & -0.031 & -0.061 & -0.110 & -0.030 & 0.007 \\
\hline 0.071 & -0.010 & & & & -0.004 & 0.098 & 0.181 & -0.005 & 0.047 & 0.039 & 0.008 & 0.032 & 0.079 & -0.010 & 0.048 & 0.029 \\
\hline 0.076 & 0.046 & & & & 0.039 & 0.177 & 0.180 & 0.048 & 0.075 & 0.087 & 0.022 & 0.092 & 0.203 & 0.064 & 0.105 & 0.047 \\
\hline-0.049 & -0.027 & 0.055 & 0.008 & -0.153 & & & & -0.029 & -0.047 & -0.046 & -0.022 & -0.048 & -0.090 & -0.030 & -0.056 & -0.035 \\
\hline 0.023 & 0.011 & -0.038 & 0.006 & 0.059 & & & & 0.012 & 0.021 & 0.021 & 0.008 & 0.023 & 0.045 & 0.012 & 0.027 & 0.016 \\
\hline 0.027 & 0.016 & -0.017 & -0.014 & 0.094 & & & & 0.017 & 0.025 & 0.025 & 0.014 & 0.026 & 0.046 & 0.018 & 0.029 & 0.019 \\
\hline-0.031 & -0.005 & 0.042 & -0.017 & -0.069 & -0.012 & -0.036 & -0.039 & & & & -0.010 & -0.022 & -0.039 & 0.001 & -0.028 & -0.020 \\
\hline 0.014 & 0.007 & -0.026 & 0.018 & 0.043 & 0.008 & 0.021 & 0.024 & & & & 0.007 & 0.015 & 0.021 & 0.000 & 0.020 & 0.012 \\
\hline 0.017 & -0.002 & -0.017 & -0.001 & 0.026 & 0.004 & 0.015 & 0.015 & & & & 0.003 & 0.007 & 0.018 & -0.001 & 0.008 & 0.008 \\
\hline-0.044 & -0.022 & 0.072 & -0.023 & -0.083 & -0.022 & -0.079 & -0.084 & -0.023 & -0.042 & -0.042 & & & & -0.018 & -0.053 & -0.031 \\
\hline 0.012 & 0.008 & -0.017 & 0.012 & 0.019 & 0.008 & 0.021 & 0.022 & 0.008 & 0.014 & 0.012 & & & & 0.004 & 0.016 & 0.010 \\
\hline 0.032 & 0.014 & -0.055 & 0.011 & 0.064 & 0.014 & 0.058 & 0.062 & 0.015 & 0.029 & 0.030 & & & & 0.014 & 0.037 & 0.021 \\
\hline-0.022 & 0.004 & 0.085 & -0.058 & -0.048 & -0.005 & -0.023 & -0.020 & 0.004 & -0.018 & -0.013 & -0.002 & -0.014 & -0.025 & & & \\
\hline 0.020 & 0.007 & -0.048 & 0.016 & 0.047 & 0.009 & 0.036 & 0.038 & 0.006 & 0.022 & 0.017 & 0.005 & 0.021 & 0.039 & & & \\
\hline
\end{tabular}


(IJACSA) International Journal of Advanced Computer Science and Applications,

Vol. 4, No. 10, 2013

\begin{tabular}{|c|c|c|c|c|c|c|c|c|c|c|c|c|c|c|c|c|}
\hline 0.002 & -0.011 & -0.038 & 0.042 & 0.001 & -0.003 & -0.013 & -0.019 & -0.009 & -0.005 & -0.004 & -0.003 & -0.007 & -0.014 & & & \\
\hline 0.074 & -0.054 & -0.014 & 0.000 & 0.011 & 0.003 & -0.007 & -0.025 & -0.021 & -0.030 & 0.054 & 0.005 & -0.016 & 0.003 & 0.006 & -0.040 & 0.022 \\
\hline-0.082 & 0.060 & 0.016 & 0.001 & -0.012 & -0.004 & 0.018 & 0.019 & 0.051 & -0.018 & -0.039 & 0.004 & -0.005 & 0.002 & 0.095 & -0.030 & -0.029 \\
\hline 0.008 & -0.007 & -0.002 & 0.000 & 0.001 & 0.000 & -0.011 & 0.006 & -0.030 & 0.047 & -0.015 & -0.009 & 0.021 & -0.005 & -0.101 & 0.070 & 0.007 \\
\hline-0.015 & -0.015 & -0.004 & 0.006 & -0.033 & 0.026 & -0.144 & -0.185 & -0.076 & -0.002 & 0.045 & 0.031 & -0.060 & -0.107 & -0.136 & -0.038 & 0.065 \\
\hline 0.015 & 0.015 & 0.004 & -0.006 & 0.033 & -0.026 & 0.144 & 0.185 & 0.076 & 0.002 & -0.045 & -0.031 & 0.060 & 0.107 & 0.136 & 0.038 & -0.065 \\
\hline 0.031 & 0.034 & 0.048 & 0.037 & 0.016 & 0.063 & -0.071 & -0.087 & 0.033 & 0.035 & 0.028 & 0.023 & 0.066 & 0.008 & 0.058 & 0.037 & 0.013 \\
\hline-0.031 & -0.034 & -0.048 & -0.037 & -0.016 & -0.063 & 0.071 & 0.087 & -0.033 & -0.035 & -0.028 & -0.023 & -0.066 & -0.008 & -0.058 & -0.037 & -0.013 \\
\hline 0.029 & 0.031 & 0.038 & 0.035 & 0.022 & 0.046 & -0.049 & 0.003 & 0.063 & 0.005 & 0.018 & 0.021 & 0.069 & 0.000 & 0.001 & 0.028 & 0.048 \\
\hline-0.029 & -0.031 & -0.038 & -0.035 & -0.022 & -0.046 & 0.049 & -0.003 & -0.063 & -0.005 & -0.018 & -0.021 & -0.069 & 0.000 & -0.001 & -0.028 & -0.048 \\
\hline 0.083 & 0.078 & 0.053 & 0.069 & 0.105 & 0.046 & 0.161 & 0.262 & 0.122 & 0.071 & 0.045 & 0.036 & 0.096 & 0.213 & 0.084 & 0.103 & 0.066 \\
\hline-0.083 & -0.078 & -0.053 & -0.069 & -0.105 & -0.046 & -0.161 & -0.262 & -0.122 & -0.071 & -0.045 & -0.036 & -0.096 & -0.213 & -0.084 & -0.103 & -0.066 \\
\hline-0.022 & -0.018 & -0.007 & -0.021 & -0.028 & -0.020 & 0.014 & -0.055 & 0.034 & -0.059 & -0.042 & -0.015 & -0.011 & -0.048 & 0.021 & -0.030 & -0.035 \\
\hline 0.022 & 0.018 & 0.007 & 0.021 & 0.028 & 0.020 & -0.014 & 0.055 & -0.034 & 0.059 & 0.042 & 0.015 & 0.011 & 0.048 & -0.021 & 0.030 & 0.035 \\
\hline 0.084 & 0.083 & 0.070 & 0.063 & 0.105 & 0.043 & 0.206 & 0.260 & 0.097 & 0.120 & 0.030 & 0.050 & 0.080 & 0.210 & 0.216 & 0.086 & 0.011 \\
\hline-0.084 & -0.083 & -0.070 & -0.063 & -0.105 & -0.043 & -0.206 & -0.260 & -0.097 & -0.120 & -0.030 & -0.050 & -0.080 & -0.210 & -0.216 & -0.086 & -0.011 \\
\hline 0.089 & 0.086 & 0.070 & 0.076 & 0.107 & 0.061 & 0.144 & 0.244 & 0.113 & 0.063 & 0.083 & 0.057 & 0.089 & 0.192 & 0.112 & 0.105 & 0.063 \\
\hline-0.089 & -0.086 & -0.070 & -0.076 & -0.107 & -0.061 & -0.144 & -0.244 & -0.113 & -0.063 & -0.083 & -0.057 & -0.089 & -0.192 & -0.112 & -0.105 & -0.063 \\
\hline
\end{tabular}

\begin{tabular}{|c|c|c|c|c|c|c|c|c|c|c|c|c|c|c|c|}
\hline \multicolumn{2}{|c|}{ Extroversion } & \multicolumn{2}{|c|}{ Facebook } & \multicolumn{2}{|c|}{ mixi } & \multicolumn{2}{|c|}{ Twitter } & \multicolumn{2}{|c|}{ Google+ } & \multicolumn{2}{|c|}{ You Tube } & \multicolumn{2}{|c|}{ Mobage } & \multicolumn{2}{|c|}{ Ameba } \\
\hline Outdoor & Indoor & Use & Not & Use & Not & Use & Not & Use & Not & Use & Not & Use & Not & Use & Not \\
\hline 0.011 & -0.014 & 0.000 & -0.001 & 0.000 & 0.000 & 0.000 & 0.000 & -0.002 & 0.000 & 0.000 & 0.000 & -0.002 & 0.000 & -0.001 & 0.000 \\
\hline-0.011 & 0.014 & 0.000 & 0.001 & 0.000 & 0.000 & 0.000 & 0.000 & 0.002 & 0.000 & 0.000 & 0.000 & 0.002 & 0.000 & 0.001 & 0.000 \\
\hline-0.056 & 0.072 & 0.003 & -0.005 & 0.004 & -0.004 & 0.002 & -0.002 & -0.014 & 0.004 & 0.003 & -0.003 & -0.012 & 0.003 & -0.010 & 0.003 \\
\hline 0.046 & -0.059 & -0.002 & 0.003 & -0.003 & 0.003 & -0.002 & 0.001 & 0.011 & -0.003 & -0.002 & 0.002 & 0.008 & -0.002 & 0.007 & -0.002 \\
\hline 0.010 & -0.012 & -0.001 & 0.002 & -0.001 & 0.001 & -0.001 & 0.000 & 0.004 & -0.001 & -0.001 & 0.001 & 0.004 & -0.001 & 0.003 & -0.001 \\
\hline-0.028 & 0.041 & 0.003 & 0.002 & 0.004 & 0.000 & 0.003 & 0.002 & -0.004 & 0.004 & 0.004 & 0.000 & 0.000 & 0.003 & -0.001 & 0.003 \\
\hline-0.007 & -0.051 & -0.024 & -0.030 & -0.027 & -0.026 & -0.026 & -0.027 & -0.029 & -0.026 & -0.027 & -0.026 & -0.033 & -0.025 & -0.029 & -0.026 \\
\hline 0.005 & 0.000 & 0.002 & 0.004 & 0.002 & 0.003 & 0.002 & 0.003 & 0.005 & 0.002 & 0.002 & 0.003 & 0.005 & 0.002 & 0.004 & 0.002 \\
\hline 0.009 & -0.001 & 0.004 & 0.006 & 0.004 & 0.005 & 0.005 & 0.005 & 0.007 & 0.004 & 0.005 & 0.005 & 0.007 & 0.004 & 0.007 & 0.004 \\
\hline 0.011 & 0.008 & 0.009 & 0.011 & 0.009 & 0.011 & 0.009 & 0.010 & 0.013 & 0.009 & 0.009 & 0.010 & 0.013 & 0.009 & 0.012 & 0.009 \\
\hline 0.009 & 0.003 & 0.006 & 0.007 & 0.006 & 0.006 & 0.006 & 0.006 & 0.008 & 0.006 & 0.006 & 0.006 & 0.008 & 0.006 & 0.007 & 0.006 \\
\hline 0.094 & -0.135 & -0.006 & -0.006 & -0.008 & -0.004 & -0.007 & -0.005 & 0.000 & -0.008 & -0.008 & -0.004 & -0.003 & -0.007 & -0.002 & -0.007 \\
\hline-0.094 & 0.135 & 0.006 & 0.006 & 0.008 & 0.004 & 0.007 & 0.005 & 0.000 & 0.008 & 0.008 & 0.004 & 0.003 & 0.007 & 0.002 & 0.007 \\
\hline-0.074 & -0.045 & -0.056 & -0.072 & -0.053 & -0.071 & -0.057 & -0.066 & -0.099 & -0.051 & -0.055 & -0.070 & -0.086 & -0.056 & -0.085 & -0.055 \\
\hline-0.032 & -0.033 & -0.020 & -0.052 & -0.029 & -0.036 & -0.028 & -0.036 & -0.052 & -0.027 & -0.034 & -0.031 & -0.070 & -0.023 & -0.050 & -0.027 \\
\hline 0.034 & 0.015 & 0.020 & 0.035 & 0.019 & 0.033 & 0.022 & 0.029 & 0.047 & 0.019 & 0.022 & 0.030 & 0.047 & 0.020 & 0.041 & 0.021 \\
\hline 0.072 & 0.063 & 0.057 & 0.089 & 0.063 & 0.075 & 0.064 & 0.073 & 0.104 & 0.059 & 0.067 & 0.072 & 0.110 & 0.059 & 0.095 & 0.061 \\
\hline-0.030 & -0.054 & 0.012 & -0.124 & 0.005 & -0.090 & -0.012 & -0.062 & -0.165 & -0.004 & -0.040 & -0.040 & -0.200 & 0.000 & -0.132 & -0.012 \\
\hline 0.015 & 0.026 & -0.007 & 0.058 & -0.005 & 0.043 & -0.004 & 0.036 & 0.064 & 0.005 & 0.025 & 0.010 & 0.091 & 0.000 & 0.046 & 0.009 \\
\hline 0.015 & 0.029 & -0.005 & 0.066 & 0.000 & 0.047 & 0.016 & 0.027 & 0.102 & 0.000 & 0.015 & 0.030 & 0.109 & 0.001 & 0.086 & 0.002 \\
\hline-0.043 & 0.051 & -0.054 & 0.041 & -0.017 & -0.018 & 0.009 & -0.039 & 0.046 & -0.037 & 0.019 & -0.061 & 0.008 & -0.024 & 0.022 & -0.030 \\
\hline-0.020 & -0.012 & 0.018 & 0.001 & 0.014 & 0.009 & -0.007 & 0.027 & -0.003 & 0.016 & -0.013 & 0.040 & 0.070 & -0.003 & -0.023 & 0.022 \\
\hline 0.062 & -0.040 & 0.036 & -0.042 & 0.004 & 0.009 & -0.001 & 0.013 & -0.043 & 0.021 & -0.006 & 0.021 & -0.078 & 0.027 & 0.001 & 0.008 \\
\hline-0.024 & -0.030 & 0.008 & -0.104 & -0.045 & -0.024 & -0.047 & -0.025 & -0.151 & -0.001 & -0.030 & -0.041 & -0.133 & -0.011 & -0.108 & -0.012 \\
\hline-0.002 & 0.004 & -0.009 & 0.042 & 0.028 & -0.008 & 0.034 & -0.008 & 0.027 & 0.006 & 0.015 & 0.006 & 0.005 & 0.012 & 0.012 & 0.010 \\
\hline 0.026 & 0.026 & 0.001 & 0.062 & 0.017 & 0.032 & 0.013 & 0.033 & 0.124 & -0.005 & 0.015 & 0.035 & 0.128 & -0.002 & 0.096 & 0.002 \\
\hline-0.002 & 0.078 & -0.057 & 0.068 & 0.003 & -0.022 & -0.024 & 0.004 & -0.007 & -0.009 & 0.010 & -0.031 & 0.150 & -0.049 & 0.017 & -0.017 \\
\hline-0.024 & -0.022 & 0.004 & 0.032 & 0.019 & 0.010 & 0.013 & 0.016 & 0.044 & 0.006 & 0.008 & 0.022 & 0.017 & 0.014 & 0.036 & 0.008 \\
\hline 0.027 & -0.056 & 0.052 & -0.100 & -0.022 & 0.012 & 0.012 & -0.020 & -0.037 & 0.003 & -0.019 & 0.009 & -0.167 & 0.035 & -0.053 & 0.009 \\
\hline & & 0.004 & -0.009 & -0.001 & 0.000 & -0.001 & -0.001 & -0.009 & 0.002 & -0.001 & 0.000 & -0.013 & 0.002 & -0.004 & 0.000 \\
\hline & & -0.009 & 0.017 & 0.001 & 0.001 & 0.000 & 0.002 & 0.008 & -0.002 & 0.006 & -0.005 & 0.026 & -0.006 & 0.008 & -0.002 \\
\hline & & 0.005 & -0.008 & 0.000 & 0.000 & 0.001 & -0.001 & 0.000 & 0.000 & -0.005 & 0.005 & -0.013 & 0.004 & -0.004 & 0.001 \\
\hline-0.005 & -0.038 & & & -0.010 & -0.021 & -0.010 & -0.019 & -0.064 & -0.001 & -0.021 & -0.009 & -0.091 & 0.005 & -0.053 & -0.003 \\
\hline
\end{tabular}


(IJACSA) International Journal of Advanced Computer Science and Applications, Vol. 4, No. 10, 2013

\begin{tabular}{|c|c|c|c|c|c|c|c|c|c|c|c|c|c|c|c|}
\hline 0.005 & 0.038 & & & 0.010 & 0.021 & 0.010 & 0.019 & 0.064 & 0.001 & 0.021 & 0.009 & 0.091 & -0.005 & 0.053 & 0.003 \\
\hline 0.032 & 0.032 & 0.036 & 0.024 & & & 0.037 & 0.029 & 0.014 & 0.037 & 0.033 & 0.031 & 0.012 & 0.036 & 0.019 & 0.036 \\
\hline-0.032 & -0.032 & -0.036 & -0.024 & & & -0.037 & -0.029 & -0.014 & -0.037 & -0.033 & -0.031 & -0.012 & -0.036 & -0.019 & -0.036 \\
\hline 0.030 & 0.028 & 0.034 & 0.023 & 0.034 & 0.026 & & & 0.024 & 0.032 & 0.031 & 0.029 & 0.012 & 0.035 & 0.024 & 0.032 \\
\hline-0.030 & -0.028 & -0.034 & -0.023 & -0.034 & -0.026 & & & -0.024 & -0.032 & -0.031 & -0.029 & -0.012 & -0.035 & -0.024 & -0.032 \\
\hline 0.075 & 0.090 & 0.063 & 0.109 & 0.074 & 0.089 & 0.078 & 0.084 & & & 0.082 & 0.081 & 0.133 & 0.069 & 0.113 & 0.071 \\
\hline-0.075 & -0.090 & -0.063 & -0.109 & -0.074 & -0.089 & -0.078 & -0.084 & & & -0.082 & -0.081 & -0.133 & -0.069 & -0.113 & -0.071 \\
\hline-0.021 & -0.010 & -0.025 & -0.013 & -0.019 & -0.021 & -0.019 & -0.021 & -0.019 & -0.020 & & & -0.014 & -0.021 & -0.018 & -0.021 \\
\hline 0.021 & 0.010 & 0.025 & 0.013 & 0.019 & 0.021 & 0.019 & 0.021 & 0.019 & 0.020 & & & 0.014 & 0.021 & 0.018 & 0.021 \\
\hline 0.076 & 0.104 & 0.057 & 0.126 & 0.078 & 0.091 & 0.075 & 0.091 & 0.128 & 0.071 & 0.086 & 0.081 & & & 0.119 & 0.073 \\
\hline-0.076 & -0.104 & -0.057 & -0.126 & -0.078 & -0.091 & -0.075 & -0.091 & -0.128 & -0.071 & -0.086 & -0.081 & & & -0.119 & -0.073 \\
\hline 0.085 & 0.095 & 0.073 & 0.111 & 0.083 & 0.094 & 0.085 & 0.091 & 0.122 & 0.078 & 0.089 & 0.087 & 0.130 & 0.078 & & \\
\hline-0.085 & -0.095 & -0.073 & -0.111 & -0.083 & -0.094 & -0.085 & -0.091 & -0.122 & -0.078 & -0.089 & -0.087 & -0.130 & -0.078 & & \\
\hline
\end{tabular}

